\title{
RNA-seq and metabolome revealed counter effect of chitosan against Botrytis cinerea on grape berries
}

\section{tariq pervaiz ( $\nabla$ tariq_bac@yahoo.com )}

Nanjing Agricultural University Nanjing China

\section{Haifeng Jia}

Nanjing Agricultural University

\section{Peian Zhang}

Nanjing Agricultural University

\section{Muhammad Salman Haider}

Nanjing Agricultural University

\section{Suwen Lu}

Nanjing Agricultural University

\section{Xinke Wei}

Nanjing Agricultural University

Haoran Jia

Nanjing Agricultural University

Jinggui Fang

Nanjing Agricultural University

\section{Research article}

Keywords: Botrytis cinerea, Chitosan, Growth elicitor, Transcriptomic, Metabolome, Antioxidants

Posted Date: October 11th, 2019

DOl: https://doi.org/10.21203/rs.2.15853/v1

License: (a) (i) This work is licensed under a Creative Commons Attribution 4.0 International License.

Read Full License 


\section{Abstract}

Background: Plants have great potential to protect against biotic and abiotic stresses. Hence, the interaction between defense signaling networks is incredible, which can either be activated with the application of growth elicitors or antimicrobial organic compounds.

Results: In this study, chitosan $(15 \mathrm{kDa})$ is used against grey mold (Botrytis cinerea) in two grape varieties (Shine-Muscat and Kyoho). The findings depicted that the interaction of DEGs and KEGGs in control and treated samples of grapevine, which provides the evidence for selection of gray mold defense responsive genes and chitosan for subsequent application in grapevine production/postharvest. The genes encoding cyclic nucleotide gated ion channels (CNGCs) and CaM/CML expressed a large number of transcripts, meanwhile, in treated samples, CaM/CML and RPS2 showed the highest number of up-regulated genes. In plant hormone signal transduction pathway, treated samples AUXIAA and SAUR again the highest number of transcripts were found. In correlation with metabolome analysis, 20 differentially expressed metabolites were recorded. In the negative correlation in the control samples of Kyoho vs Shine mascate showed 224 and 157 up and down-regulated respectively. Moreover, antioxidants were also significantly regulated with the chitosan application and reduced the lesion diameter, water loss and disease incidence up to 12 days. In both varieties, the chitosan treatment superoxide dismutase, peroxidase, Malondialdehyde, catalase, and proline content was significantly increased during storage.

Conclusion: The results depicted that at gene expression levels was varied at different fruit growth developmental stages, and the most effective in case of plant-pathogen interaction. Chitosan is seen to be more effective in both varieties and it acts as an anti-fungal agent. The transcriptomic study also confirmed that at the transcriptome level expression was higher in treated samples, however in general transcription factor have not much affected with chitosan application.

\section{Background}

Vitis vinifera $\mathrm{L}$. is an important fruit crop, having more than $7.5 \mathrm{mha}$ of the area under cultivation, and with $73.3 \mathrm{mt}$ production worldwide in 2017 worldwide [1]. The external influence caused by pathogens, diseases, and environment depict substantial effects on grapevine yield and productivity $[2,3]$. Plants can array positive complex molecular and physiological approaches to mitigate and cope the severity of biotic stresses [2] and disease susceptibility or resistance against disease, which not only dependents on the specific plant-pathogen interaction but also on the tissue-specific growth stages. The external influence caused by pathogens, diseases, and environment depict substantial effects on grapevine yield and productivity $[3,4]$. The ripening and maturation of fleshy and pome fruits developmental conversion that accords with susceptible to pathogenicity. Fruit ripening and maturation comprised of complex regulatory growth networks and hormone-mediated pathways leading to significant changes in the biochemical and physiological characteristics of grape berries [5]. Hemibiotrophs pathogens are important and fetal to fruits that modified lifestyles at different developmental stages and in several 
ecological conditions [6]. Thus, the infection strategies of different pathogens challenge the competency of the host to respond naturally and develop effective defense mechanisms.

Grey mold or Botrytis bunch rot caused by Botrytis cinerea is the most devastating and economically important diseases in $V$. vinifera all over the globe. Floral and immature berry tissues can be infested by $B$. cinerea and the pathogenicity can persist during semi-dormant saprophytic state in necrotic floral tissues or in a dormant state within the developing fruits $[7,8]$. Structure-activity reports the application of purified chitosan oligomers have presented that the degree of acetylation and polymerization affect their bioactivity $[9,10]$. Chitosan is a natural polysaccharide containing $\mathrm{N}$-acetylglucosamine and glucosamine copolymers.. Chitosan is also useful for the human body as a dietary supplement for subsiding body weight and decreasing the cholesterol level $[11,12]$. Chitosan is used as an effective biochemical remedy against fungal attack during storage and replaced the $\mathrm{SO}_{2}$ treatments, resulting in the control of fungal diseases $[13,14]$. Chitosan with low molecular weight compounds are the most effective against fungal and defensive reactions than with the higher molecular weight. Furthermore, it has been reported to develop systemic resistance in plants. Oligosaccharide growth elicitors from various pathogens reported having a critical role in host plant protection mechanism encompassing plant pathogen chemoperception chemoperception and interaction [15].

The omic technologies: transcriptomic, genomics, proteomics and metabolomics are focused on genes, RNA, proteins, and metabolite [16]. RNA-sequencing is novel and deep-sequencing NGS technique used to acquire transcriptomic profiling. In a single assay, the method reveals the discovery of transcript information, allele-specific, unique genes, single nucleotide variants and gene expression without the accessibility of ESTs annotations. Furthermore, transcriptomic files have also been used in defining largescale genes controlling the complex pathways and interaction and metabolic activities of plants during different environmental conditions $[4,17]$. Consequently, metabolomics allow the investigation of metabolites that are existing in higher concentration, mainly major some important carboxylic acids, amino acids, and sugars. Thus, the significant roles of many metabolites but less concentrated metabolites during growth and development is still need to investigate [16].

To take a further step to investigate the grapevine defense responses against fungus, metabolome and transcriptome were examined. Due to the fast modification in techniques and advancement in technology of NGS including metabolomicmetabolomics, genomic, ionomic, proteomic, transcriptomic, phenomic, the producing quantity of sequencing data in experiments has increased. Due to the application of this technology leads to an improved level of transcriptome revelation, enriched the sequencing reads mapping. Considerably, constant developments in defining the Vitis transcriptome in functional annotation $[18,19]$ and GOs consignments [20], currently it permits the detailed description of the functional assignment of $V$. vinifera genes [21]. So far there are very few reports predicted on gene expression of chitosan effects; though, first unreported facts from the transcriptomic investigation of the chitosan Colletotrichum on avocado cv. Hass collaboration indicates important modifications in the gene expression levels of the host and pathogen relation [22]. Therefore, keeping in the above techniques and problem the comprehensive study of comparative transcriptome and metabolomic analysis is design to 
understand the responses of chitosan and identify putative genes, metabolites and their role in defense and metabolic pathways.

\section{Results And Discussion}

\section{Scanning Electron Microscopy Samples}

The berry samples treated with chitosan , mycelial growth of Botrytis cinerea was disordered, the overall growth rate slowed down, most of them collapsed, no longer obvious growth, a large number of branches appeared at the growing point, the branch spacing became shorter, deformity appeared, showing swelling or candidal-shaped, and exudation of inclusions. However, the Botrytis cinerea without chitosan treatment grew well on the surface of fruits and mycelia were slender, straight, uniform in thickness and smooth in line. The surface of mycelia was smooth, and its branches were formed at a certain distance from the top of mycelia, and the growth point was thin and well extended (Fig. 2).

\section{Sequence quality control and Illumina RNA-seq}

The data retrieved showed that the total raw reads in Kyoho control KC1 showed highest 52,864,680 in all three controls followed by clean reads 51,534,216 and clean bases 7.73G. However in Shine-muscat control showed $60,211,220$ raw reads, $59,144,494$ clean reads and $8.87 \mathrm{G}$ clean bases, which is also higher than the Kyoho control. In a comparison of treated samples, Kyoho KT1 showed highest raw reads $67,165,372$, clean reads $65,368,848$ followed by $9.81 \mathrm{G}$ clean bases. Meanwhile, Shine-muscat ST2 has highest raw reads 59,068,078, with 58,381,062 clean reads, and $8.76 \mathrm{G}$ clean bases. in overview, KT1 expressed a higher number of raw reads, clean reads and clean bases (Table 2). The results showed that the expression level in KT will be higher than the control and Shine-muscat. This also may be due to varietal effect with the chitosan treatment and cellular activities of RNA during metabolism and fruit development.

In case of total mapped reads in control Shine-muscat SC1 have higher percentage i.e. 53,045,265 (89.69\%) and in comparison within treatments Kyoho KT1 generated 57,982,396 (88.7\%) total mapped reads, followed by ST R2, 52,585,277 (90.07\%). Moreover 2,727,030 (4.94\%) multiple mapped reads showed in SC2, which is higher as compared to all treatments and varieties. However uniquely mapped reads were generated $55,414,067$ (84.77\%) by Kyoho KT1, followed by SC1 50,738,565 (85.79\%) (Table $3)$.

Furthermore, total differential expressed genes (DEG) in KC vs SC are 12,693 (6,854 up and 5,839 downregulated), in KT vs KC 9,788 (4,535 up and 5,253 down-regulated), KT vs ST 8,495 (3,905 up and 4,590 down-regulated) and in ST vs SC 5058 (3012 up and 2046 down-regulated) were recorded. The FPKM hierarchical clustering map, log10 (FPKM+1) values are normalized (scale number) and clustered, red indicates high expression gene, blue indicates low expression gene. The color ranges from red to blue, indicating log10 (FPKM+1) from large to small; log2 (ratios) line graph, the gray line in each sub-graph indicates the relative expression of the gene in a cluster under different experimental conditions (Fig. 3 A). 
DEGs were generating a Venn diagram of up and down-regulated genes. The differential genes in Venn diagram show the number of differential expressed genes between the comparison groups and the overlap between the comparison groups. It shows the number of DEGs per comparison in control of both varieties and treated once, and the overlap between each set of genes shows the total up and downregulated genes. 2,166 genes were commonly expressed in all samples including treated and control of both varieties. The total numbers of genes in KT vs KC showed 9788 , ST vs SC 5058, KC vs SC $12,693, \mathrm{KT}$ vs ST 8495 transcripts (log2-fold change of $1, \mathrm{P}>0.05$ ) that were differentially regulated are shown (Fig. 3 $B$ and $C)$.

\section{GO and KEGG Enrichment Analysis of Differential Expressed Gene}

To detect the functions of Genes expression involved in the response to chitosan application, we determined GO assignments. Differentially expressed genes enrichment analysis in GOs were carried out by the GOseq R package software, where gene length bias were corrected. GO terms with modified P.value considered as significantly enriched, which will be $<0.05$ by differential expressed genes. [23]. Maximum number of DEGs were generated in biological process (BP), followed by cellular component (CC) and the rest of them in molecular function (MF) categories in all treated and control samples. In comparisonwith control and treatment, the KC vs SC has a high value of GO terms i.e 4,341 followed by KT vs KC 4,180 (Fig.4). However, in both varieties, Kyoho and Shine-muscat treated samples assigned 3,967. Meanwhile up-regulated GOs were higher in treated samples and lower in control.. In case of treated samples (highest in KT vs KC enriched with 5,595/15,822), significantly enriched terms were biological regulation, cellular processes, and metabolic process, hormonal regulation, plant-pathogen interaction, and these terms are more important to gray mold and disease incident. In cellular component group (highest showed in KC vs SC 4,139/8,869 followed by KT vs KC 3,275/8,869), significantly enriched terms included organelle part, cell, extracellular regions, cell part, proteinaceous extract, and apoplast, which were found. In the molecular function category (KC vs SC 8,472/19,544 enriched followed by KT vs KC $6,663 / 19,544)$, significantly enriched terms included transcription factor, transporter activity, transcription factor activity, coenzymes binding, catalytic, binding, nucleic acid binding and transducer activity (Fig. 4).

Kyoto Encyclopedia of Genes and Genomes (KEGG) is a system for examining gene function and genomic information databases, which is helpful to investigate gene expressions in complete pathway network. As a huge public database related to Pathways [24], KEGG provides excellent integration of developmental pathway networks, including biodegradation of organic matter, metabolism of nucleosides, carbohydrates, and amino acids. The most enrichment significant pathways analysis of DEGs, allow to in-depth knowledge in the KEGG database. This analyses led to expedite understanding the genes interactions and biological functions $[25,26]$. The database has useful information for thoughtful functions and use of the biological system, such as from molecular-level information, especially large-scale molecular datasets generated by high-throughput and genome sequencing experimental technologies, organism and the ecosystem (http://www.genome.jp/kegg/). In present study the $K C$ vs SC group, DEGs were significantly enriched in five pathways with $Q$ and $P$-values $<0.05$ : "Biosynthesis of secondary metabolites" (591 DEGs), "Plant hormone signal transduction" (132 DEGs), 
"Plant-pathogen interaction" (150), Transcription factor (21) and protein export (36) were enriched. Meanwhile, in treated samples KT vs ST Biosynthesis of secondary metabolites" (483 DEGs), "Plant hormone signal transduction” (89 DEGs), "Plant-pathogen interaction” (127) and Transcription factor (8) and protein export (18) were enriched. The enrichment factor is the ratio of the number of DEG to the background number in a different pathway. In comparison between controls of both varieties had shown in Figure 5. The results depicted that KC and SC enriched in RNA transport, plant-pathogen interaction, carbon metabolism and biosynthesis of Amino acid. The pathways DEGs enrichment is changed as after treated with growth elicitor chitosan. The enrichment factor ratio in KT vs ST showed higher in metabolic pathways, plant-pathogen interaction and purine metabolism (Fig.5).

\section{Genes Expressed in Plant Pathogen Interaction Pathway}

The interaction of plants with pathogens influenced programmed cell death (PCD) pathways. During a resistance interaction, PCD was induced in a process termed the hypersensitive response (HR) which may function to limit pathogen spread [27]. Against pathogen outbreak, crops must have the potential to induce particular defense proteins. Cell walls play a critical role as barriers that can inhibit the outburst of pathogens, but also have an important stock of energy-glucose [28]. The samples analyzed in control (KC vs SC) for plant-pathogen interaction pathway 150 DEGs were recorded. In fungal PAMP the CDPK showed two up-regulated and eight transcripts were down-regulated, in the same Rboh had two up and single down-regulated genes. Meanwhile no gen was detected in ROS (Supplementary File S1). In the case of Bacterial flagellin signaling (flg22), FLS2 showed one down-regulated, and MEKK1 has two up and two down-regulated. In the same series MKK1/2, MKK4/ 5 and MKK3/6 showed one up-regulated each. Furthermore in case of Bacterial EF-Tu two up-regulated and one down-regulated. Moreover, in Bacterial Secretion system, PBS2 and RPM1 showed highest transcripts, four up and four down-regulated and ten up-regulated and four down-regulated respectively. However, in HSP90 have a shown very different number of transcripts that is two up and four down-regulated. However in Kyoho treated vs control (KT vs KC) the larger number of transcripts in Bacterial Secretion system (RPS2) fifteen were upregulated and three showed down-regulation, followed by in CDPK five up and five down-regulated (S2).

The treated sample of both varieties was compared for plant-pathogen interaction, KT vs ST in Fungal PAMP, CDPK expressed two up and four down-regulated and Rboh have a single down-regulated transcript. Fungal PAMP ( $\mathrm{CaMCML}$ ) have a correlation with $\mathrm{Ca}$ and also showed six up and seven downregulated. In the case of Bacterial Flagellin flg22, MEKK1 has two up and single down-regulated transcripts (S2). Meanwhile MKK1/2, MKK4/5, and MPK3/6, single up-regulated transcript was recorded in each. In the same pathway Bacterial EF-Tu have one up and three down-regulated. Furthermore, in Bacterial secretion system RPM1 and RPS2 expressed four up and seven down-regulated, and sixteen up and twenty-three down-regulated respectively. However, HSP90 have all up-regulated (4) transcripts. In the comparison of control and treated, ST vs SC CDPK showed single up and two down-regulated, Rboh have single up and CsMCML have single up and sixteen down-regulated genes were depicted. Meanwhile in the same comparison Bacterial Secretion system RPM1 showed four up and single down-regulated, RPS2 showed three up and ten down-regulated. Furthermore in HSP9O have five down-regulated transcripts 
were recorded. In cases of Bacterial flagellin flg22, FLS2, MEKK1, MEDKK1/2 did not expressed any transcript, only in BAKEKKI have four up-regulated, MEKK4/5 have single up-regulated, WRKY288 have two and PR1 have single up-regulated transcripts were recorded (S2).

\section{Plant hormone signal transduction in relation to chitosan}

Plant hormones play a crucial role in controlling the disease attack, growth, and development of plants. The hormonal regulation haste the development of the specific organ and assimilate them to produce the form in plants. Its impotent to investigate that how hormones are synthesized and processed, how they act at both the molecular and organismal levels, a description of some of the roles they play in regulating plant metabolism, and the projections for the genetic engineering of hormone production levels or reactions in plants protection $[29,30]$. In the recent study, hormonal singling pathways many genes were expressed in control (KC vs SC), mainly in Tryptophan metabolism, AUX1 showed two down and one upregulated, TIR1 have two up and one down-regulated, in the same series AUXIAA at two points expressed thirteen number of transcripts, out of which twelve are down and one is up-regulated, followed by GH3 four and one up and down-regulated respectively. However SAUR have very divergent from rest of the hormone expression (eleven up and six down-regulated). In the second Brassinosteroid biosynthesis, BAK1 and BSK expressed two up and one down-regulated each, in the same way, BIN2 Proteasomal degradation showed two up-regulation, BZR1/2 and CYCD3 have one down-regulated each, and finally $\mathrm{TCH} 4$ have four down-regulated transcripts (S3). Another important stage of a-Linolenic Acid metabolism but they didn't have any such remarkable transcript regulation, only single transcript at each stage is down-regulated, furthermore in biosynthesis of Phenylalanine TGA have shown three up and one downregulated. In Kyoho (KT vs KC) Tryptophan Metabolism, AUX1 showed three up and single downregulated TIR1 single up and two down-regulate and in AUXIAA have seven up and three down-regulated, while SAUR showed three up and eight down-regulated genes. In Zeatin Biosynthesis CRE1, AHP and BARR have two, single and five up-regulated transcripts were observed respectively. Large numbers of transcripts were recorded in Carotenoid biosynthesis, in PYR/PYL have two up and one down-regulated, PP2C five up and two down-regulated. SnRK2 four up and single down-regulated, ABF showed five up and single down-regulated transcripts were recorded (S3).

In treated samples of shine-muscat and Kyoho (KT vs ST) Plant Hormone single transduction pathway, Tryptophan metabolism expressed three up-regulated in AUX1, one up and two down-regulated in TIR1, four up and seven down-regulated in AUXIAA followed by six up and six down-regulated by SAUR.. In Zeatin biosynthesis is also different from control, where all transcripts are up-regulated in CRE1, AHP, and B-ARR (2 up-regulated in each), but in Brassinosteroid biosynthesis, BAK1 have single up and four downregulated transcripts were recorded. In a-Linolenic Acid metabolism, all transcripts in JAR1and COI1 are up-regulated, meanwhile in Phenylalanine metabolism NPR1 have all up-regulated and TGA have one up and two down-regulated transcripts were recorded. In comparison with ST vs SC Tryptophan Metabolism, AUXIAA showed two up and eleven down-regulated, followed by SAUR five up and five down-regulated. In case of Brassinosteroid Biosynthesis, in BAK1 four up-regulated, BSK two up and one down-regulated. 
However, in BZR1/2 and TCH4 showed four down-regulated in each. Meanwhile, in Phenylalanine metabolism, TGA has three and PR-1 single up-regulated (S3).

\section{Biosynthesis of secondary metabolites and interaction with Chitosan application}

Syntheses of secondary metabolites in response to growth elicitors are important during growth and organ development. An analysis of pathways generally and stage specific secondary metabolites were highly regulated in early developmental phase [25]. In the control samples, total down-regulated transcripts are 264 and up-regulated are 345, and in treated (KT vs ST) samples 132 up and 324 transcripts were down-regulated. The more relevant and important secondary metabolites are Biotin metabolism, Arginine and proline metabolism, Tryptophan, Terpenes, Zeatin, Alkaloids, Glycolysis / Gluconeogenesis were regulated in treated samples and during growth phases.

The number of transcripts in control KC vs SC three secondary metabolites were up-regulated during Biotin metabolism, twenty-eight in Arginine and proline metabolism, seven in tryptophan, ten in diterpenoid, eight in zeatin, ten in isoquinoline alkaloid and forty-three in glycolysis / gluconeogenesis metabolism. While seven down-regulated transcripts in biotin metabolism, twenty-three in arginine and proline metabolism, thirteen in tryptophan, three in diterpenoid, nine in isoquinoline alkaloid and fortyeight during glycolysis/gluconeogenesis metabolism.

Furthermore, in treated samples (KT vs ST), secondary metabolites recorded as; one in biotin metabolism, sixteen in arginine and proline metabolism, five in tryptophan, diterpenoid, four in zeatin, three in isoquinoline alkaloid and thirteen in glycolysis / gluconeogenesis are metabolism. Meanwhile, seven down-regulated in biotin, twenty-five in Arginine and proline metabolism, nine in tryptophan, four in diterpenoid, one in zeatin, thirteen in isoquinoline alkaloid and glycolysis/gluconeogenesis fifty-one transcripts are recorded.

\section{Basal transcription factors pathway in association with defense}

Transcripts during transcription factors pathway in control of grapes were recorded, in KC vs SC general transcription factors for RNA polymerase II have shown number of transcripts, in TFIIB expressed one up and two down-regulated, in TFIID, TAF1TAF 10, TAF12 showed single down-regulation transcript. Meanwhile TAF5 TAF6, TAF13 have single gene with up-regulation. In addition TFIIH, TFIIH1, TFIIH4 and MAT1 have single down-regulated genes, while in TTDA and CDK7 have one and two up-regulated transcripts respectively (S4). In the comparison of treatment and control KT vs KC, the General transcription factors for RNA Polymerase II showed very few transcripts expression as compared to control, such as TFIIA2, TAF5, TAF7, TAF13, TFIIF2, TFIIH, TFIIH1, CDK7, and MAT1 showed no expression. TFIIB, TFIID, TAF1, TAF7, TAF5, TAF6, TAF4, TAF10, TAF13, TFIIE, TFIIE1, TFIIF, TFIIF1, TFIIF2, TFIIH, TFIIH1 and TFIIH4 expressed single up-regulated transcripts, however only TTDA showed single down-regulated transcripts (S4). 
In comparison of KT vs ST, in general transcription factors for RNA polymerase II have single up and down-regulated in TFIIB, one is up-regulated in TAF8, TAF5, and TTDA, while in CDK two up-regulated and TFIIH1 have one down-regulated transcripts were found. As in comparison with ST and SC the number of transcripts were reduced, only TFIIB one up and TAF8 have one down-regulated transcripts were recorded (S4). However, over the transcription expression was very low and less number of transcripts were found in control as well as in treated samples. This shows that the pathogen attack and general transcription factors did not show any close regulation at any stage of berry infestation.

\section{Co-regulated gene analysis between transcriptome and metabolome}

Pathways related to metabolic activities were more stimulated in treated samples. Secondary metabolites and terpenoid biosynthesis (sesquiterpenoid, triterpenoid, and diterpenoid, biosynthesis), phenylpropanoid (flavonol flavone, and phenylpropanoid biosynthesis) were highly enriched transcripts with higher expression levels (Fig. 6,7, S1)

Correlation analysis, and comparison of metabolites and genes were recorded in the results of genetic and metabolic correlation analysis. 20 differential metabolites and 50 differential genes were expressed. In the KC vs SC showed negative correlation with 224 up and 157 down-regulated genes as shown in Table 4. Furthermore 6 genes in Purine metabolism, 5 in Biosynthesis of secondary metabolites, with 3 each in Histidine metabolism and Flavone and flavonol biosynthesis. However in positive correlation were observed in the same comparison Glucosinolate biosynthesis, 2-0xocarboxylic acid metabolism, Glycine, serine and threonine metabolism, methionine and cysteine metabolism, Pantothenate and CoA biosynthesis, Glycerophospholipid metabolism, Tryptophan metabolism, tyrosine and tryptophan, Phenylalanine, biosynthesis, Histidine metabolism, Purine metabolism showed single genes in all metabolome (Fig. 7, S5). In positive correlation, KC vs SC 830 genes were up-regulated. Moreover in treated samples 730 transcripts were down-regulated (Table 3). Isoquinoline alkaloid biosynthesis, Fructose and mannose metabolism showed single genes. Meanwhile in KT vs ST positive correlation depicted 638 up and 1069 down-regulated. Monoterpenoid biosynthesis, Glucosinolate biosynthesis, 2-0xocarboxylic acid metabolism, Glycine, serine and threonine metabolism, Glycerophospholipid metabolism, Zeatin biosynthesis, Tryptophan metabolism, Phenylalanine, tyrosine and tryptophan biosynthesis, Pyrimidine metabolism, Cysteine and methionine metabolism, ABC transporters, Plant hormone signal transduction, alpha-Linolenic acid metabolism, Glutathione metabolism, Metabolic pathways and Biosynthesis of unsaturated fatty acids, positively regulated (single gene) (Fig. 6, S5). In negatively regulated genes 131 up and 207 down-regulated (Table 3). These co-regulated genes were also presented in heatmap. The redder the color, the stronger the positive correlation, the bluer the color, and the stronger the negative correlation presented in the heatmap (Fig. 7, S5).

DEGs Validation by qRT-PCR: For reproducibility and validation of data generated from Illumina sequence technique, thirteen transcripts from RNA-Seq randomly selected representing the entire major up and down-regulated functions were selected. These selected transcripts were involved in plant-pathogen interactions, hormone signaling, and seconder metabolic activities. The results of qRT-PCR for all the 13 
(7 up and 6 down-regulated) were selected genes and results are consistent with the RNA-Seq results, underscoring the accuracy. The data reliability of Illumina sequence, qRT-PCR primers are presented in Supplementary S1 and Fig. 8.

\section{Physiological and Antioxidant regulation in response to chitosan}

The application of chitosan on grape berries showed a positive impact on the regulation of antioxidants with the time lapse. The results showed that with the increase in time period and infection the antioxidants activities were higher as shown in Figure 9 and 10. In case Kyoho, SOD in treated samples were increased as compared with control up to 12 days (i.e. $0.81 \mathrm{U} / \mathrm{mg}$ protein), POD also showed increasing trend with the increase in time, and after 12 days the value was $71.7 \mathrm{U} / \mathrm{mg}$ as compared with control i.e. 42.7, CAT was also increased up to $8.5 \mathrm{u} / \mathrm{mg}$. Proline content was increased up to $722 \mathrm{ng} / \mathrm{g}$, $\mathrm{O}_{2} 45 \mathrm{nmol} / \mathrm{mg}$ and MDA was $7.32 \mathrm{nmol} / \mathrm{g}$. However, in Shine-Muscat, the antioxidant trend was almost higher than the Kyoho, for SOD $2.33 \mathrm{U} / \mathrm{mg}$, which was more than Kyoho. POD $91.2 \mathrm{U} / \mathrm{mg}$ and CAT was $10.5 \mathrm{U} / \mathrm{mg}$, proline content $937 \mathrm{ng} / \mathrm{g}$ and reactive $\mathrm{O}_{2} 38 \mathrm{nmol} / \mathrm{mg}$ min protein, which is lower than Kyoho, MDA $4.96 \mathrm{nmol} / \mathrm{g}$, is almost half than Kyoho and higher than control (Fig. 9).

The fruits samples were also assessed for physiological parameters, in which a significant difference was observed as compared to control. In Kyoho berries fruit samples fruit firmness was $5.8 \mathrm{~N}$, water loss rate $98.495 \%$, disease index $11.9 \%$, berry abscission incidence $13.6 \%$ was recorded. However, disease incidence $20.8 \%$, lesion diameter $3.7 \%$ was decreased and lower than control i.e. $23.5 \%$ and lignin content $4.98 \%$ was recorded (Fig. 10 ). In case of Shine-muscat fruit firmness was $8.8 \mathrm{~N}$, water loss rate $98.181 \%$, and disease index $6.7 \%$, berry abscission incidence $4.7 \%$, disease incidence $13.2 \%$, lesion diameter $1.7 \%$ and lignin content $5.87 \%$ was recorded (Fig.10).

\section{Discussion}

Although the research on resistance to $B$. cinerea has been carried out extensively, in postharvest fruits is relatively few. Generally, Sulfur dioxide fumigation is used to treat grapes to prevent disease infection, but excessive sulfur is harmful to human body, and is prone to sulfur pollution, resulting in the decline of grape quality after harvest. Chitosan, a polysaccharide polymer and harmless to human body,have been reported to reduce $B$. cinerea during pre-harvest and postharvest treatments, and to modulate selected defense genes[31, 32]. In pathways signal transduction produced by transcription factors, which could activate a number of genes expression, with the subsequent physiological events, frequently connecting changes in cell-wall structure, ion fluxes, de novo production of synthesis of phytoalexins, pathogenesisrelated (PR) and reactive oxygen species (ROS) proteins production [33, 34]. However, the signaling pathways regulate comprehensive resistance and several component networks with typical schemes (Pieterse, 2009). Grapes are widely grown in the world, it plays an important role in people's life, therefore, postharvest disease resistance and fresh-keeping are becoming more and more important to realize the 
economic value of grapes. The studies of molecular mechanism of chitosan in induction of grape fruit resistant against $B$. cinerea infection was very useful for chitosan extensive application and improvement of application measures. To elucidate the whole transcriptional changes with the chitosan in grapes, following pre-harvest application of growth elicitors to the berries, transcriptome data were generated and validated by RT-qPCR. In the transcriptome, we found many disease-resistance genes changes after chitosan application, and the MeJA biosynthesis genes, such as AOS, OPR3, were increased. MeJA is a important disease-resistance factor during fruit development, we propose that chitosan induced MeJA production in resistance against $B$. cinerea. Meantime, metabolomics analysis showed that many substances were changed after chitosan application that responded to $B$. cinerea infection. We found a lot of metabolism, in transcriptome-metabolomics association analysis, were affected from genes expression to metabolism production, suggesting that chitosan indeed influenced many disease resistance signal pathway in protection grape fruit from $B$. cinerea infection. These above is in accord with that the chitosan decreased the $B$. cinerea infection compared to the water treatment, and the ShineMuscat grape had more resistance against the B.cinerea, suggesting that chitosan could protect the grape from disease infection in molecular and physiological levels. Therefore, the molecular mechanism of chitosan in resistance to B.cinerea was very important for revealing the effects of chitosan in protection grape from disease infection.

After screening differential genes enrichment analysis was conducted to study the distribution of differential genes in Gene Ontology in order to clarify the expression of gene differences in the experiments (Fig.4). The principle of ordinary GO enrichment analysis is hypergeometric distribution. The hypergeometric distribution relationship between these differential genes and some specific branches in the GO classification is calculated according to the selected differential genes, and a specific $p$-value is obtained through hypothesis verification, either the differential gene is rich in the GO. Enrichment analyses depicted that the involvement of GOs were higher in treated samples and lower in control, as well as in control vs treatment. In case of treated with chitosan the BP group (highest in KT vs KC enriched with 5,595/15,822), significantly enriched terms were biological regulation, cellular processes, and metabolic process, hormonal regulation and plant-pathogen interaction, and these terms are more important to gray mold and disease incident (Fig 4). In the previous reports tomato and Arabidopsis transcriptomic changes in response to $B$.cinerea stress, and also examine the expression profiles of $B$. cinerea-inoculated plants to examine the defense transcriptome and reported genes involved in plant responses to the pathogenicity[35]. In cellular component group (highest showed in KC vs SC 4,139/8,869 followed by KT vs KC 3275/8869), significantly enriched terms included organelle part, cell, extracellular regions, cell part, proteinaceous extract, and apoplast were found. In the molecular function category (KC vs SC 8472/19544 enriched followed by KT vs KC 6663/19544), significantly enriched terms included transcription factor, transporter activity, transcription factor activity, coenzymes binding, catalytic, binding, nucleic acid binding and transducer activity. RNAseq data and GO analysis exposed plasma membrane, oxidoreductase activity, and transport as main categories induced [36]. Chitosan also enriches transport GO functions, respiration and oxidative metabolism in the model yeast Saccharomyces cerevisiae[37]. 
The samples analyzed in control (KC vs SC) for pathogen interaction pathway, the total of $150 \mathrm{DEGs}$ were recorded. In fungal PAMP, the CDPK showed two up-regulated and 8 down-regulated transcripts. However in case of treated samples the up-regulated transcripts were same, but the down-regulated were reduced (4). This shows the interaction of in fungal PAMP the CDPK have a potential role in inducing resistance in berries (S1), T Boller and G Felix [38] also highlighted the importance of PAMPs, which are perceived by the plant, the basis for the first offense of active crop immunity, termed as PAMP-triggered immunity (PTI). As their role expected from plant-pathogen interactions, 14-3-3 proteins undertake substantial changes succeeding PAMP sensitivity. During the response against fungal and insect attack PAMP chitosan 14-3-3 genes were reported in poplar and in sycamore cell cultures, PAMP induces accumulation of 14-3-3 proteins [39, 40]. Evidently, in A. thaliana 14-3-3k is the closest homolog in tomato TFT1, which is a need for PAMP-triggered accumulation of ROS, a reaction intimidated by HopM1. Hence, it has been wondered that HopM1 suppresses in primary PTI responses, specifically PAMP-triggered accumulation of ROS and stomatal closure, through the decline of 14-3-3k/TFT1, however the direct link to be investigated. The facts gathered from various pathosystems and crop species, this suggested that a conspicuous function of 14-3-3 proteins in immunity and defense [41, 42].. In case of KT vs KC, the numbers of up-regulated transcripts were decreased and the down-regulation is more then up-regulated transcripts in FLS2, and same as in case of KV vs ST down-regulated is more then up-regulated expression (S1). FLS2 plays a key role in the flg22-mediated stimulation against defense through the MAPK cascade. Anyhow the total detection of flgll-28 appears inadequate to a subset of species in the Solanaceae family, complementary with flg22, which is generally documented across the plant kingdom [45-47]. Plants that showed a deficiency of FLS2 are more vulnerable to Pseudomonas attack, these findings showed that FLS2 can function at an initial growth to interfere with bacterial access into the apoplast $[48,49]$.

In the case of control the Tryptophan Metabolism, showed more down-regulated genes i.e. in AUX1 (1), TIR1 (2), AUXIAA (11), ARF (3), GH3 (1), SAUR (6). While in comparison with treatment the expression pattern was changed with chitosan treatment, namely in AUX1 (3), AUXIAA (6), and SAUR (6) transcripts up-regulated. This shows that the effect of chitosan on fruit will change the expression levels (S2). In treated and a control sample of Shine-muscat, Tryptophan Metabolism showed large number of genes down-regualted in case of AUXIAA (11), ARF (1), GH3 (1), SAUR (5), as same in treated samples (S2).In fungal species, plant-pathogens produce chitosan throughout early infection to elude host defenses, in endophyte Pestalotiopsis sp. CDA produces chitosan oligomers that different in its chitin substrates no longer provoke plant resistance[50, 51][52]. The nematophagous fungus Pochonia chlamydosporia accumulate chitosan through Meloidogyne javanica egg infection. Overexpresses of chitosanase and CDA encoding genes during this process in fungs. The action of the catalytic center of a CDA encoded in the Pochonia chlamydosporia genome recently documented and proven experimentally [53, 54]. As early reported by F Lopez-Moya, M Suarez-Fernandez and LV Lopez-Llorca [54], chitosan is one of plant defense modulator, and encourages Auxin production in $A$. thaliana roots. This polymer is sources of overexpression in the biosynthetic pathway of tryptophan-dependent auxin. It also able to blocks auxin translocation in the root system. Furthermore, some fungal and bacterial pathogens accumulate CKs. 
more alike Auxs. CKs suppress resistance reactions (such as HR) to Pseudomonas savastanoi, chitosan application also stimulated both callose apposition and ABA accumulated in leaf tissues $[55,56]$.

The most relevant secondary metabolites were regulated by chitosan in treated samples with the higher expression, as like in case of Biotin metabolism, Arginine and proline metabolism, Tryptophan, Terpenes, Zeatin, Alkaloids, Glycolysis / Gluconeogenesis synthesis. The capacity of Antioxidant in fruit was increased, the expression pattern is directly or indirectly reliant on the level of flavonoid classes, such as anthocyanins and flavonols [57]. Improved health benefits of fruit can be acquired since eminent levels of secondary metabolites. Induction of gene expression in strawberries related to growth and overproduction of secondary metabolites is the important objective for the future study. The use of synthetic chitosan/agrochemicals will enhance the quality and quantity of fruit and higher contents of secondary metabolites. Chitosan application enhanced growth and also triggered the accumulation of phenolic secondary metabolites in fruits [58]. Y He, S Bose, W Wang, X Jia, $\mathrm{H} \mathrm{Lu}$ and $\mathrm{H}$ Yin [59] also suggested that the chitosan application will not only trigger plant immunity, but also influences the accumulation of several secondary metabolites. Furthermore, chitosan also improved the cell wall structure and controlled gene expression of specific significant enzymes convoluted in cell wall degradation and ethylene compound biosynthesis.

In the present study, we observed the clear difference of expressions in control and treated samples in General Transcription factors, mainly in TFIID (TAF7, TAF5, TAF6, TAF13), which showed most of upregulated transcripts as compared to the treated sample. However, in comparison with KT vs KC () the expression levels were higher (TFIIB, TAF1, TAF6, TAF4, TAF10, TFIIH4), than only treated samples (S3). As predictable, the metabolites involved in metabolic pathways also showed different expression levels. The gene-specific transcription factors binding leads to the conscription of adaptor complexes mainly Mediator or SAGA, both of which in turn expedites binding of general transcription factors (GTFs). In the precious investigation, it is clear that chitosan act as growth elicitor and antifungal activities to synthesizing multiple Transcription factor (TFIIB) is an important constituent of the RNA polymerase II instigation complex. TFIIB transmits out two major tasks: i.e. helps to recruit RNA polymerase II into the initiation complex and it cooperates directly with the TATA-binding protein. The sequence of TFIIB reveals a budding zinc-binding domain and a defective repetition of almost seventy amino acids [60]. Initially was a fast and later on steady Cin5p-mediated response. Transcription factor Cin5p/Yap4p intricate in responses against various stresses. CIN5 deletion led to improved chitosan sensitivity [61]. The GTFs (TFIIA, TFIIB, TFIID, TFIIE, TFIIF, and TFIIH) each plays a specific function. e.g., TFIIA and TFIIB are responsible for the locating of Pol II to the promoter [62]. The identified transcripts in chitosan regulation during fungal attack at biochemical, molecular, cellular level are presented. The results also depicted the co-regulated genes in metabolome and transcriptional changes during the gray mold attach and chitosan application in fruits.

In the present study, we also found the antioxidant activates are regulated by chitosan and with the increase in time the SOD, CAT, POD, and purine activities was significantly higher in both varieties. Usually, ROS metabolism is regulated by a number of enzymes mostly including POD, APX, CAT, and SOD. 
$\mathrm{H}_{2} \mathrm{O}_{2}$ is also destroyed primarily by CAT and APX. It was documented fact that the application of chemical can increase in H2O2 content is mediated by a reticence of CAT and APX in many crops [63]. This evidence led to the importance of chitosan against the attack of gray mold on berries and plays as a barrier and increases the pathogen incidence.

\section{Conclusion}

Presented results emphasize on In the present comparative study of metabolome and transcriptome profiling of the defense, responsive pathways. and genes were evaluated,. weWe described the control and treated grape berries of Shine-muscat and Kyoho against $B$. cinereagray mold attack. The results depicted that at gene level expression was vary at different levels, and the most effective in case of plantpathogen interaction, Chitosan is seen to be more effective in both varieties and it acts as an anti-fungal agent. The transcriptomic study also confirmed that at the transcriptome level expression was higher in treated samples as compared to control, however in general transcription factor have not much affected with chitosan application. There is a need to more in-depth investigation of genes and genes profiling. The identified transcripts in this study will be more helpful to understand the mechanism in chitosan regulation during fungal attack at biochemical, molecular, cellular level. The results also depicted the coregulated genes in metabolome and transcriptional changes during the gray mold attach and chitosan application in fruitsfruits. (Fig. 10) and differentially expressed transcripts were validated via qrtPCR technique (Fig. 10).

\section{Methods}

\section{Grape berry treatment with Resistance Inducers Chitosan}

Two Grape varieties Grapes (Shine-muscat and Kyoho) were gown at experimental filed field Nanjing Agricultural University, Nanjing China. Two Grape verities The samples were treated with resistance inducers chitosan (15 kDa), the physiological changes in the grape berry were analyzed at $80-90 \%$ physiologically maturities, with commercial grade chitosan, and formulations were applied by foliar spray: $15 \%$ (w/v) chitosan (15 kDa) (15\% (w/v) chitosan 15 kDa Fig.1). To prepare

formulationsFormulations were prepared with, we used distilled water as a control. Each berry of grapy is treated as one replication and each replication is repeated together and randomized 3 times. One day later, Botrytis cinerea spores with $10^{4} / \mu$ l concentration were usedapplied. The gray mold fungus was grown on grape berries at $25{ }^{\circ} \mathrm{C}$, and with $95 \%$ relative humidity. Spores were washed from the fruit followed by 15 days with distilled water with $0.01 \%$ Tween 20 . Later on, a number of spores are added to the inoculation solution at the proper concentration. The control and chitosan-treated grape fruits were incubated in inoculation solution which containing $10^{4}$ ssporesspores in $1 \mu \mathrm{l}$ for $5 \mathrm{~min}$. Then the grape berries were incubated on paper at $25{ }^{\circ} \mathrm{C}$ under $95 \%$ relative humidity. Relative humidity (95-100\%) was maintained by covered with plastic film. The Infection infestation status was checkedtested by counting the total number of lesions on each fruit surface on a daily bases. Counting of biomass on the grape 
fruits (Table 1). Antioxidants were extracted from For extraction of antioxidants, $0.5 \mathrm{~g}$ of berries were and crushed into $5 \mathrm{ml}$ of $50 \mathrm{~mm}$ phosphate buffer with in an ice bath. After centrifuging supernatant was extracted and homogenate for 20 mints at $18,928 \mathrm{~g}\left(4^{\circ} \mathrm{C}\right)$, which was used for assays of enzyme activities as outlined as described by S Verma and R Dubey [64]. The activities of antioxidant enzymes were quantified by the following methods mention by Haider et al., [65]. Aftertreated with Botrytis cinerea we collected samples for each treatment and control (Fig.1), and frozen in liquid nitrogen, followed by deposited at $-80^{\circ} \mathrm{C}$ for further RNA-seq and experimental use.

\section{Sample Preparation of for Scanning Electron Microscope Microscopy Samples}

The samples was were treated by 0"Connell 's method: the sample was fixed at room temperature with glutaraldehyde of $3 \%-4 \%$ volume fraction for $4-6$ hours, washed with phosphate buffer (PBS, pH 6.8) for 4-6 times at intervals of 20-30 minutes, then dehydrated gradient with series of acetone (volume fraction $30 \%, 50 \%, 70 \%, 80 \%, 90 \%, 95 \%$, and $100 \%$ ) at intervals of 30 minutes, of which $100 \%$ acetone dehydrated three times and pure acetic acid. Isoamyl ester was replaced twice, 30 minutes each time. The critical point of $\mathrm{CO}_{2}$ was dry, sticky and coated. The samples were observed and photographed under JSM-6360LV scanning electron microscope[66].

\section{RNA Extraction, cDNA library construction, and Illumina deep Seq}

Total RNA was extracted from three biological fruit replicates, all samples including each treatment and control; we also used control for both spartanly separately as for Kyoho and Shine-muscat. RNA extraction was done by using Trizol (Invitrogen, Carlsbad, CA, USA) reagent method and consequently recycled for mRNA refinement and library construction with the Ultra ${ }^{\mathrm{TM}}$ RNA Library Prep Kit for Illumina (NEB, USA) following the manufacturer's instructions. The treated and control samples were sequenced on an Illumina Hiseq ${ }^{\text {TM }} 2000$. Sequencing was completed by the Beijing Nuohe Zhiyuan Technology Co., Ltd. Beijing, China. After the sample was tested, the mRNA was enriched by binding of the A-T complementary pairing to the clone A tail of the mRNA using magnetic beads with Oligo (dT). Subsequently, the fragmentation buffer was used to break the mRNA into short fragments. Using mRNA as a template, a single-strand cDNA was synthesized using random hexamers, and then double-stranded cDNA was synthesized by adding buffer, dNTPs and DNA polymerase I, followed by AMPure. XP beads purified double-stranded cDNA. The purified double-stranded cDNA was subjected to terminal repair, A tail was added and the sequencing linker was ligated, and then AMPure XP beads were used for fragment size selection, and finally, PCR enrichment was performed to obtain a final cDNA library. The raw image data files obtained by high-throughput sequencing (Illumina HiSeqTM) are converted into the original sequencing sequence by CASAVA Base Calling analysis. We call it Raw Data or Raw Reads, and the result is FASTQ. 
The sequences reads were mapped to a grape reference genome (ftp://ftp.ensemblgenomes.org/pub/plants/release-32/fasta/vitis_vinifera/) for further analysis using TopHat v2.0.9 and Bowtie. To functionally annotate genes, the unique sequences were annotated against public databases, including the National Center for Biotechnology Informationnon-redundantprotein(Nr) (http://www.ncbi.nlm.nih.gov/) and Swiss-Prot (http://www.expasy.ch/sprot/), Gene Ontology (GO), and Kyoto Encyclopedia of Genes and Genomes (KEGG) [67] databases.

\section{Cluster Analysis}

The read numbers mapped to each gene were counted using HTSeq v0.6.17. Raw counts of the genes were normalized to the fragments per kilobase of transcript per million mapped reads (FPKM). The average FPKM value of each gene was calculated and transformed to two as the log base. The expression patterns of all the genes both in the treatment and the control were characterized by TCseq [68].

\section{Differential Gene Expression Analysis}

The FPKM value was employed to quantify the gene expression levels. The DEseq [67] was used to identify differentially expressed genes (DEGs) from RNA-seq data. The thresholds for the significance test were set as a false discovery rate $(F D R) \leq 0.01$ and |Fold Change| $\geq 2$ [68]. The enrichment analysis of GO and KEGG were done using Cluster Profiler [68].

\section{Functional Analysis}

The hypergeometric distribution relationship between differentially expressed genes and some specific branches in the GO classification is calculated according to the selected differential genes, and a specific p-value is obtained through hypothesis verification. Whether the differential gene is enriched in the GO. Software approach used in our analysis of GO enrichment analysis is GO seq [23], which is based on the Wallenius non-central hypergeometric distribution. Compared to the ordinary hypergeometric distribution, this distribution is characterized by the fact that the probability of extracting an individual from a certain category is different from the probability of extracting an individual from outside a certain category, and the probability is different. It is estimated by estimating the length of the gene so that the probability of $\mathrm{GO}$ term being enriched by differential genes can be calculated more accurately.

Pathway Significant Enrichment Analysis Using Pathway in the KEGG database, hypergeometric tests were applied to find Pathway that was significantly enriched in differentially expressed genes compared to the entire genome background. Calculated as follows: 
Here $\mathrm{N}$ is the number of genes with Pathway annotation in all genes; $\mathrm{n}$ is the number of differentially expressed genes in $\mathrm{N} ; \mathrm{M}$ is the number of genes annotated as a particular Pathway in all genes; $\mathrm{m}$ is the number of differentially expressed genes annotated as a particular Pathway. When FDR $\leq 0.05$, it indicates that the differential gene is significantly enriched in the Pathway. Significantly enriched KEGG pathways were identified with KO-based annotation system (KOBAS) 2.0 [67], using the p-value.

\section{Extraction, Identification, and Quantification of Metabolite}

The grape berries were placed on ice, and $50 \%$ methanol buffer is used for metabolites extraction. Briefly, samples of $20 \mu \mathrm{l}$ were extracted with precooled $50 \%$ methanol of $120 \mu \mathrm{L}$, and carried out vortexed for 1 min, followed by incubation at room temperature (10 $\mathrm{min})$; after that, the extracted mixture kept overnight at $-20^{\circ} \mathrm{C}$. Aand centrifuged at $4000 \times \mathrm{g}$ for $20 \mathrm{~min}$, and supernatants were moved into new plates having 96-well as followed by the methods of Y Li, J Fang, X Qi, M Lin, Y Zhong, L Sun and W Cui [68].

LC-MS raw data into the mzXML format by using MS Convert, followed by the XCMS, metaX toolbox, and CAMERA, employed in the R software. The RT (combined retention time) and $\mathrm{m} / \mathrm{z}$ data were used to pinpoint each ion [68-70]. To elucidate the biological functions, physical and chemical properties of metabolites, the available PLANTCYC (http://www.plantcyc.org/), KEGG (http://www.kegg.jp/) and inhouse (http://spldatabase.saskatoonlibrary.ca/), HMDB (http://www.hmdb.ca/) databanks were used to perform level-one and level-two identification and annotation as described by $Y$ Li, J Fang, X Qi, M Lin, Y Zhong, L Sun and W Cui [68]. Screening and quantitative analysis for differential metabolites were conducted using metaX software (http://metax.genomics.cn/) as mentioned by [68, 71]. In order to be able to correlate with the transcription sample, first made the number of samples consistent, transcribe each comparison to 3 repetitions, and metabolize each comparison to 6 replicates, in order to eliminate Data is standardized by the magnitude of the impact.

\section{qRT-PCR}

To validate the expression patterns by qRT-PCR, we used to control and treated samples for both grape verities varieties at the developmental stages of berries. PrimeScript RT Reagent Kit with gDNA Eraser (Takara, China) is used to purify RNA samples and reverse-transcribed using the following the manufacturer's protocol. Thirteen representative random transcripts were selected for the qRT-PCR assay and carried out as described earlier by Pervaiz et al., 2016 [25]. Gene-specific primers were designed using Primer3 (http://primer3.ut.ee/) software, as mentioned in supplementary files S1.

\section{Statistical Analysis}

Statistical analysis was performed using SPSS statistic 21.0 software (IBM, Armonk, NY, USA). One-way analysis of variance $(p \leq 0.05)$ was conducted. Data are means $\pm S E$ from three independent biological replicates. 


\section{Abbreviations}

SMC: Shine-muscat control

SMT:Shine-muscat Treated

KYC:Kyoho control

KYT:Kyoho Treated

SOD:Superoxide dismutase

POD: Peroxidase

MAD: Malonaldhyde

CAT: Catalase

\section{Declarations}

\section{Conflict of interest/>Competing Interests}

There is no conflict of interest between authors

\section{Consent to publish:}

Not Applicable

\section{Ethics approval and consent to participate}

In this study dose not need any ethicl approval

\section{Data Statement}

Present data is submitted to NCBI: with accession number GSE129046:https://www.ncbi.nlm.nih.gov/geo/query/acc.cgi?acc = GSE129046

\section{Author's contribution}

Experimental design and research work carried out by: $\mathrm{HJ}, \mathrm{PZ}, \mathrm{MSH}$, Data analyzed by: $\mathrm{HJ}$ and TP, Contributed materials/analysis tools: SL, and X, Article write up: TP, HJ, JF 


\section{Acknowledgments}

This work is supported by grants from National Natural Science Foundation of China (31872938), Foundation for Distinguished Young Talents in Jiangsu (BK20180076), Fundamental Research Funds for the Central Universities $₫ K Y Z 201736 \rrbracket$

\section{References}

1.OIV: Statistical report on world vitiviniculture. 2018.

2.Haider MS, Kurjogi MM, Khalil-Ur-Rehman M, Fiaz M, Pervaiz T, Jiu S, Haifeng J, Chen W, Fang J: Grapevine immune signaling network in response to drought stress as revealed by transcriptomic analysis. Plant Physiology and Biochemistry 2017, 121:187-195.

3.Jiménez S, Dridi J, Gutiérrez D, Moret D, Irigoyen JJ, Moreno MA, Gogorcena Y: Physiological, biochemical and molecular responses in four Prunus rootstocks submitted to drought stress. Tree physiology 2013, 33(10):1061-1075.

4. Haider MS, Zhang C, Kurjogi MM, Pervaiz T, Zheng T, Zhang C, Lide C, Shangguan L, Fang J: Insights into grapevine defense response against drought as revealed by biochemical, physiological and RNA-Seq analysis. Scientific reports 2017, 7(1):13134.

5.Giovannoni JJ: Genetic regulation of fruit development and ripening. The plant cell 2004, 16(suppl 1):S170-S180.

6.Kleemann J, Rincon-Rivera LJ, Takahara H, Neumann U, van Themaat EVL, van der Does HC, Hacquard S, Stüber K, Will I, Schmalenbach W: Sequential delivery of host-induced virulence effectors by appressoria and intracellular hyphae of the phytopathogen Colletotrichum higginsianum. PLoS pathogens 2012, 8(4):e1002643.

7.Reglinski T, Elmer P, Taylor J, Wood P, Hoyte S: Inhibition of Botrytis cinerea growth and suppression of botrytis bunch rot in grapes using chitosan. Plant pathology 2010, 59(5):882-890.

8.Pezet R, Viret O, Perret C, Tabacchi R: Latency of Botrytis cinerea Pers.: Fr. and biochemical studies during growth and ripening of two grape berry cultivars, respectively susceptible and resistant to grey mould. Journal of Phytopathology 2003, 151(4):208-214.

9.Cabrera JC, Messiaen J, Cambier P, Van Cutsem P: Size, acetylation and concentration of chitooligosaccharide elicitors determine the switch from defence involving PAL activation to cell death and water peroxide production in Arabidopsis cell suspensions. Physiologia plantarum 2006, 127(1):4456 . 
10.Wang $\mathrm{Y}, \mathrm{He} \mathrm{H}$, Zhou Y: Effect of different molecular weight chitosan on several physiological and biochemical characteristics related with plant defense reaction. Plant Physiology Communications 2006, 42(6):1109.

11.Mhurchu CN, Dunshea-Mooij C, Bennett D, Rodgers A: Effect of chitosan on weight loss in overweight and obese individuals: a systematic review of randomized controlled trials. Obesity reviews 2005, $6(1): 35-42$.

12.Kao C-H, Hsiang C-Y, Ho T-Y: Assessment of chitosan-affected metabolic response by peroxisome proliferator-activated receptor bioluminescent imaging-guided transcriptomic analysis. PloS one 2012, 7(4):e34969.

13.Romanazzi G, Nigro F, Ippolito A, Divenere D, Salerno M: Effects of pre-and postharvest chitosan treatments to control storage grey mold of table grapes. Journal of Food Science 2002, 67(5):18621867.

14.Romanazzi G, Feliziani E, Baños SB, Sivakumar D: Shelf life extension of fresh fruit and vegetables by chitosan treatment. Critical reviews in food science and nutrition 2017, 57(3):579-601.

15.Li P, Linhardt R, Cao Z: Structural characterization of oligochitosan elicitor from Fusarium sambucinum and its elicitation of defensive responses in Zanthoxylum bungeanum. International Journal of Molecular Sciences 2016, 17(12):2076.

16.Pinu F: Grape and wine metabolomics to develop new insights using untargeted and targeted approaches. Fermentation 2018, 4(4):92.

17.Silva P, Gerós H: Regulation by salt of vacuolar H+-ATPase and H+-pyrophosphatase activities and $\mathrm{Na}+/ \mathrm{H}+$ exchange. Plant signaling \& behavior 2009, 4(8):718-726.

18.Pruitt KD, Tatusova T, Brown GR, Maglott DR: NCBI Reference Sequences (RefSeq): current status, new features and genome annotation policy. Nucleic acids research 2011, 40(D1):D130-D135.

19.Fortes AM, Agudelo-Romero P, Silva MS, Ali K, Sousa L, Maltese F, Choi YH, Grimplet J, MartinezZapater JM, Verpoorte R: Transcript and metabolite analysis in Trincadeira cultivar reveals novel information regarding the dynamics of grape ripening. BMC plant biology 2011, 11(1):149.

20.Du Z, Zhou X, Ling Y, Zhang Z, Su Z: agriGO: a GO analysis toolkit for the agricultural community. Nucleic acids research 2010, 38(suppl_2):W64-W70.

21.Sweetman C, Wong DC, Ford CM, Drew DP: Transcriptome analysis at four developmental stages of grape berry (Vitis vinifera cv. Shiraz) provides insights into regulated and coordinated gene expression. BMC genomics 2012, 13(1):691. 
22.Gutiérrez-Martínez P, Chacón-López A, Xoca-Orozco LA, Ramos-Guerrero A, Velázquez-Estrada R, Aguilera-Aguirre S: Chitosan and Changes in Gene Expression During Fruit-Pathogen Interaction at Postharvest Stage. In: Chitosan in the preservation of agricultural commodities. Elsevier; 2016: 299-311.

23.Young MD, Wakefield MJ, Smyth GK, Oshlack A: Gene ontology analysis for RNA-seq: accounting for selection bias. Genome biology 2010, 11(2):R14.

24.Kanehisa M, Araki M, Goto S, Hattori M, Hirakawa M, Itoh M, Katayama T, Kawashima S, Okuda S, Tokimatsu T: KEGG for linking genomes to life and the environment. Nucleic acids research 2007, 36(suppl_1):D480-D484.

25.Pervaiz T, Haifeng J, Haider MS, Cheng Z, Cui M, Wang M, Cui L, Wang X, Fang J: Transcriptomic analysis of grapevine (cv. Summer black) leaf, using the illumina platform. PloS one 2016, 11(1):e0147369.

26.Upadhyay S, Phukan UJ, Mishra S, Shukla RK: De novo leaf and root transcriptome analysis identified novel genes involved in Steroidal sapogenin biosynthesis in Asparagus racemosus. BMC genomics 2014, 15(1):746.

27.Devarenne TP, Martin GB: Manipulation of plant programmed cell death pathways during plantpathogen interactions. Plant signaling \& behavior 2007, 2(3):188-190.

28.Blanco-Ulate B, Morales-Cruz A, Amrine KC, Labavitch JM, Powell AL, Cantu D: Genome-wide transcriptional profiling of Botrytis cinerea genes targeting plant cell walls during infections of different hosts. Frontiers in plant science 2014, 5:435.

29.Davies PJ: The plant hormones: their nature, occurrence, and functions. In: Plant hormones. Springer; 2010: 1-15.

30.Doares SH, Syrovets T, Weiler EW, Ryan CA: Oligogalacturonides and chitosan activate plant defensive genes through the octadecanoid pathway. Proceedings of the National Academy of Sciences 1995, 92(10):4095-4098.

31.Feliziani E, Landi L, Romanazzi G: Preharvest treatments with chitosan and other alternatives to conventional fungicides to control postharvest decay of strawberry. Carbohydrate Polymers 2015, 132:111-117.

32.Landi L, De Miccolis Angelini RM, Pollastro S, Feliziani E, Faretra F, Romanazzi G: Global transcriptome analysis and identification of differentially expressed genes in strawberry after preharvest application of benzothiadiazole and chitosan. Frontiers in plant science 2017, 8.235.

33.Landi L, Feliziani E, Romanazzi G: Expression of defense genes in strawberry fruits treated with different resistance inducers. Journal of Agricultural and Food Chemistry 2014, 62(14):3047-3056. 
34.van Loon LC, Rep M, Pieterse CM: Significance of inducible defense-related proteins in infected plants. Annu Rev Phytopathol 2006, 44:135-162.

35.Kong W, Chen N, Liu T, Zhu J, Wang J, He X, Jin Y: Large-scale transcriptome analysis of cucumber and Botrytis cinerea during infection. PloS one 2015, 10(11):e0142221.

36.Lopez-Moya F, Kowbel D, Nueda MJ, Palma-Guerrero J, Glass NL, Lopez-Llorca LV: Neurospora crassa transcriptomics reveals oxidative stress and plasma membrane homeostasis biology genes as key targets in response to chitosan. Molecular BioSystems 2016, 12(2):391-403.

37.Jaime MD, Lopez-Llorca LV, Conesa A, Lee AY, Proctor M, Heisler LE, Gebbia M, Giaever G, Westwood JT, Nislow C: Identification of yeast genes that confer resistance to chitosan oligosaccharide (COS) using chemogenomics. BMC genomics 2012, 13(1):267.

38.Boller T, Felix G: A renaissance of elicitors: perception of microbe-associated molecular patterns and danger signals by pattern-recognition receptors. Annual review of plant biology 2009, 60:379-406.

39. Malerba M, Crosti P, Cerana R: Defense/stress responses activated by chitosan in sycamore cultured cells. Protoplasma 2012, 249(1):89-98.

40.Lapointe G, Luckevich MD, Cloutier M, Séguin A: 14-3-3 gene family in hybrid poplar and its involvement in tree defence against pathogens 1. Journal of experimental botany 2001, 52(359):13311338.

41.Lozano-Durán R, Robatzek S: 14-3-3 proteins in plant-pathogen interactions. Molecular PlantMicrobe Interactions 2015, 28(5):511-518.

42.Lozano-Durán R, Bourdais G, He SY, Robatzek S: The bacterial effector HopM1 suppresses PAMPtriggered oxidative burst and stomatal immunity. New Phytologist 2014, 202(1):259-269.

43.Liu J, Ding P, Sun T, Nitta Y, Dong O, Huang X, Yang W, Li X, Botella JR, Zhang Y: Heterotrimeric G proteins serve as a converging point in plant defense signaling activated by multiple receptor-like kinases. Plant physiology 2013, 161(4):2146-2158.

44.Liu X, Yang S, Zhao M, Luo M, Yu C-W, Chen C-Y, Tai R, Wu K: Transcriptional repression by histone deacetylases in plants. Molecular plant 2014, 7(5):764-772.

45.Clarke CR, Chinchilla D, Hind SR, Taguchi F, Miki R, Ichinose Y, Martin GB, Leman S, Felix G, Vinatzer BA: Allelic variation in two distinct Pseudomonas syringae flagellin epitopes modulates the strength of plant immune responses but not bacterial motility. New Phytologist 2013, 200(3):847-860.

46.Albert M, Jehle AK, Lipschis M, Mueller K, Zeng Y, Felix G: Regulation of cell behaviour by plant receptor kinases: Pattern recognition receptors as prototypical models. European journal of cell biology 2010, 89(2-3):200-207. 
47.Asai T, Tena G, Plotnikova J, Willmann MR, Chiu W-L, Gomez-Gomez L, Boller T, Ausubel FM, Sheen J: MAP kinase signalling cascade in Arabidopsis innate immunity. Nature 2002, 415(6875):977.

48.Zipfel C, Robatzek S, Navarro L, Oakeley EJ, Jones JD, Felix G, Boller T: Bacterial disease resistance in Arabidopsis through flagellin perception. Nature 2004, 428(6984):764.

49.Abramovitch RB, Anderson JC, Martin GB: Bacterial elicitation and evasion of plant innate immunity. Nature Reviews Molecular Cell Biology 2006, 7(8):601.

50.Sanchez-Vallet A, Mesters JR, Thomma BP: The battle for chitin recognition in plant-microbe interactions. FEMS microbiology reviews 2015, 39(2):171-183.

51.Pusztahelyi T: Chitin and chitin-related compounds in plant-fungal interactions. Mycology 2018, 9(3):189-201.

52.Cord-Landwehr S, Melcher RL, Kolkenbrock S, Moerschbacher BM: A chitin deacetylase from the endophytic fungus Pestalotiopsis sp. efficiently inactivates the elicitor activity of chitin oligomers in rice cells. Scientific reports 2016, 6.38018.

53.Aranda-Martinez A, Grifoll-Romero L, Aragunde H, Sancho-Vaello E, Biarnés X, Lopez-Llorca LV, Planas A: Expression and specificity of a chitin deacetylase from the nematophagous fungus Pochonia chlamydosporia potentially involved in pathogenicity. Scientific reports 2018, 8(1):2170.

54.Lopez-Moya F, Suarez-Fernandez M, Lopez-Llorca LV: Molecular Mechanisms of Chitosan Interactions with Fungi and Plants. International journal of molecular sciences 2019, 20(2):332.

55.Robert-Seilaniantz A, Navarro L, Bari R, Jones JD: Pathological hormone imbalances. Current opinion in plant biology 2007, 10(4):372-379.

56.Iriti M, Faoro F: Abscisic acid is involved in chitosan-induced resistance to tobacco necrosis virus (TNV). Plant Physiology and Biochemistry 2008, 46(12):1106-1111.

57. Heinonen IM, Meyer AS, Frankel EN: Antioxidant activity of berry phenolics on human low-density lipoprotein and liposome oxidation. Journal of Agricultural and Food Chemistry 1998, 46(10):4107-4112.

58.Rahman M, Mukta JA, Sabir AA, Gupta DR, Mohi-Ud-Din M, Hasanuzzaman M, Miah MG, Rahman M, Islam MT: Chitosan biopolymer promotes yield and stimulates accumulation of antioxidants in strawberry fruit. PloS one 2018, 13(9):e0203769.

59. He Y, Bose S, Wang W, Jia X, Lu H, Yin H: Pre-Harvest treatment of chitosan oligosaccharides improved strawberry fruit quality. International journal of molecular sciences 2018, 19(8):2194.

60.Buratowski S, Zhou H: Functional domains of transcription factor TFIIB. Proceedings of the National Academy of Sciences 1993, 90(12):5633-5637. 
61.Zakrzewska A, Boorsma A, Brul S, Hellingwerf KJ, Klis FM: Transcriptional response of Saccharomyces cerevisiae to the plasma membrane-perturbing compound chitosan. Eukaryotic Cell 2005, 4(4):703-715.

62.Boorsma A: Dissection of transcriptional regulation networks and prediction of gene functions in Saccharomyces cerevisiae. 2008.

63.Landberg T, Greger M: Differences in oxidative stress in heavy metal resistant and sensitive clones of Salix viminalis. Journal of Plant Physiology 2002, 159(1):69-75.

64.Verma S, Dubey R: Lead toxicity induces lipid peroxidation and alters the activities of antioxidant enzymes in growing rice plants. Plant Science 2003, 164(4):645-655.

65.Haider MS, Kurjogi MM, Khalil-ur-Rehman M, Pervez T, Songtao J, Fiaz M, Jogaiah S, Wang C, Fang J: Drought stress revealed physiological, biochemical and gene-expressional variations in 'Yoshihime'peach (Prunus Persica L) cultivar. Journal of Plant Interactions 2018, 13(1):83-90.

66.O'CONNELL R, Chen Y, Zhang H, Zhou Y, Fox D, Maguire P, Wang JJ, Rodenburg C: Comparative study of image contrast in scanning electron microscope and helium ion microscope. Journal of microscopy 2017, 268(3):313-320.

67.Xie C, Mao X, Huang J, Ding Y, Wu J, Dong S, Kong L, Gao G, Li C-Y, Wei L: KOBAS 2.0: a web server for annotation and identification of enriched pathways and diseases. Nucleic acids research 2011, 39(suppl_2):W316-W322.

68.Li Y, Fang J, Qi X, Lin M, Zhong Y, Sun L, Cui W: Combined Analysis of the Fruit Metabolome and Transcriptome Reveals Candidate Genes Involved in Flavonoid Biosynthesis in Actinidia arguta. International journal of molecular sciences 2018, 19(5):1471.

69.Kuhl C, Tautenhahn R, Bottcher C, Larson TR, Neumann S: CAMERA: an integrated strategy for compound spectra extraction and annotation of liquid chromatography/mass spectrometry data sets. Analytical chemistry 2011, 84(1):283-289.

70.Wen B, Mei Z, Zeng C, Liu S: metaX: a flexible and comprehensive software for processing metabolomics data. BMC bioinformatics 2017, 18(1):183.

71.Khan N, Bano A, Rahman MA, Rathinasabapathi B, Babar MA: UPLC-HRMS-based untargeted metabolic profiling reveals changes in chickpea (Cicer arietinum) metabolome following long-term drought stress. Plant, cell \& environment 2019, 42(1):115-132.

\section{Figures}




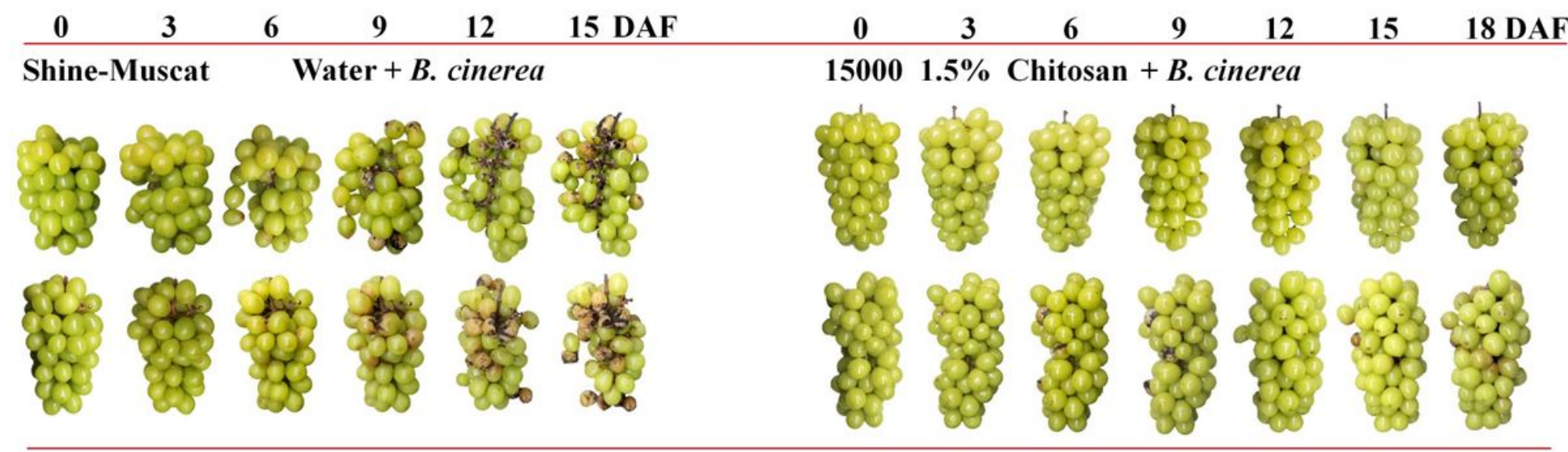

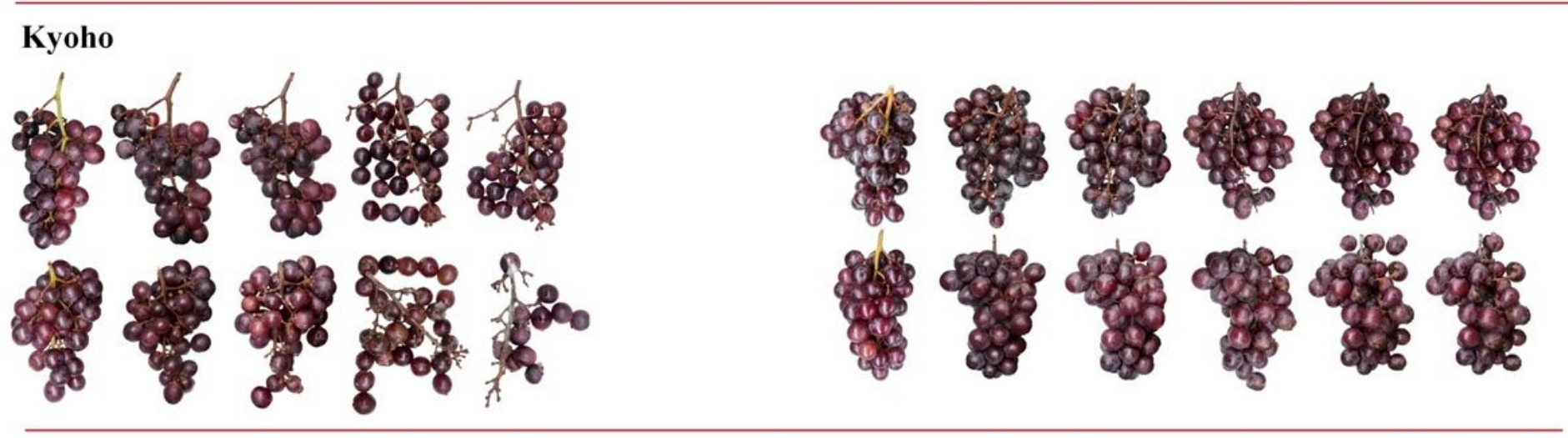

Figure 1

Grape berries of Shine-muscat and Kyoho (treated and control) with the application and days of chitosan treatment 

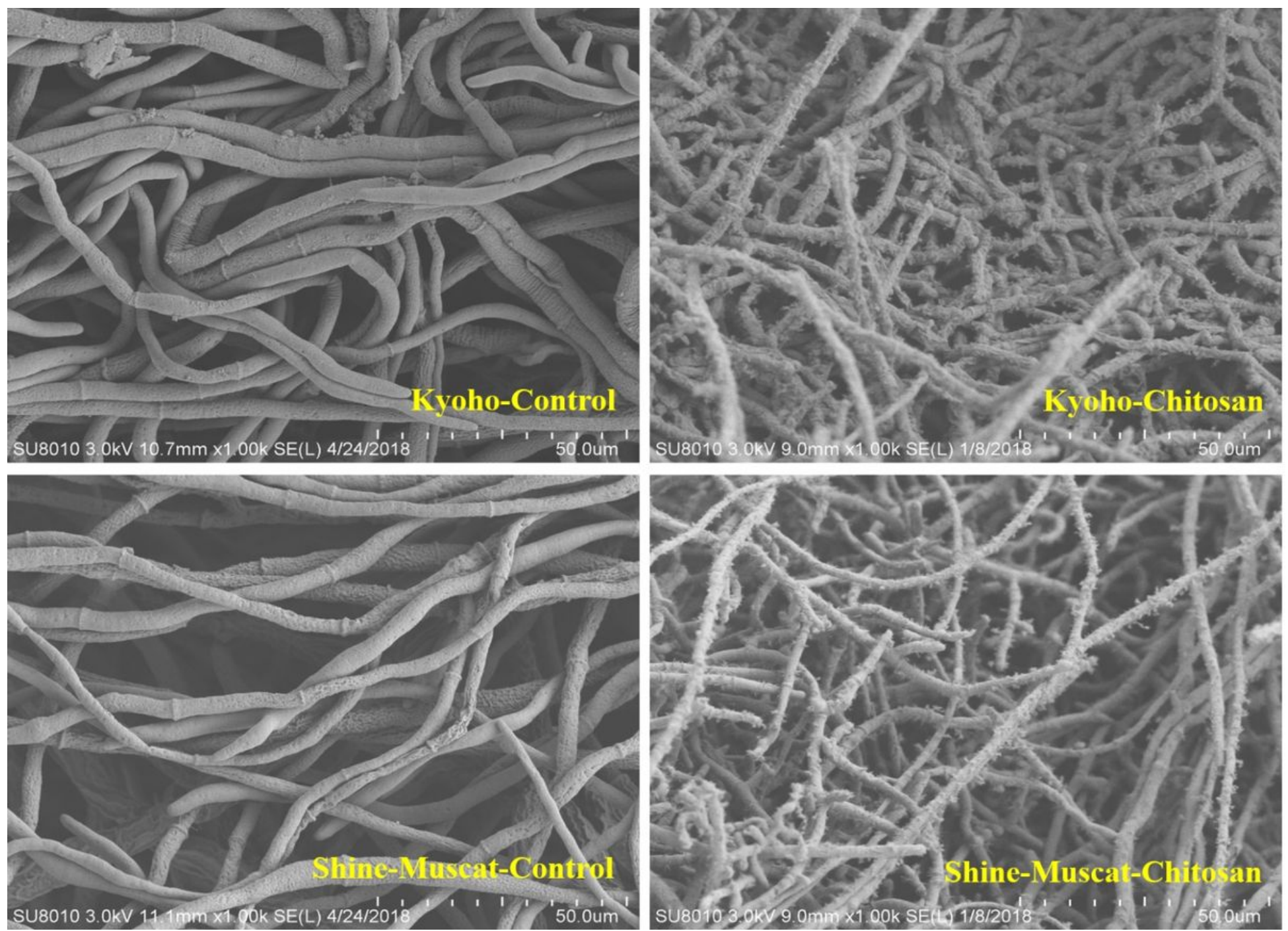

Figure 2

Effect of chitosan treatment on mycelial morphology of grape Botrytis cinerea 
A

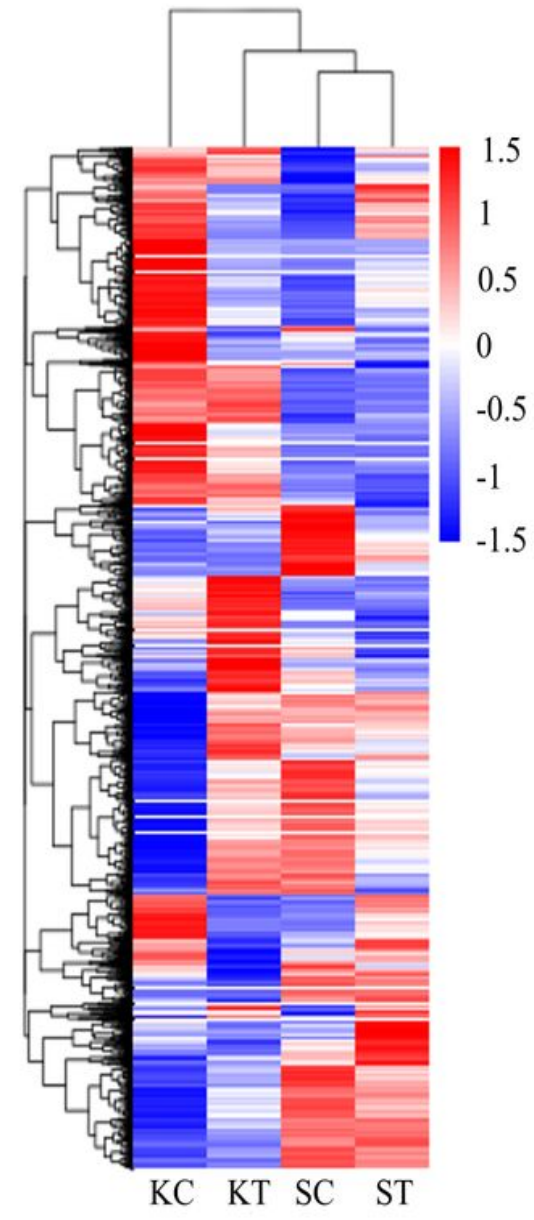

B

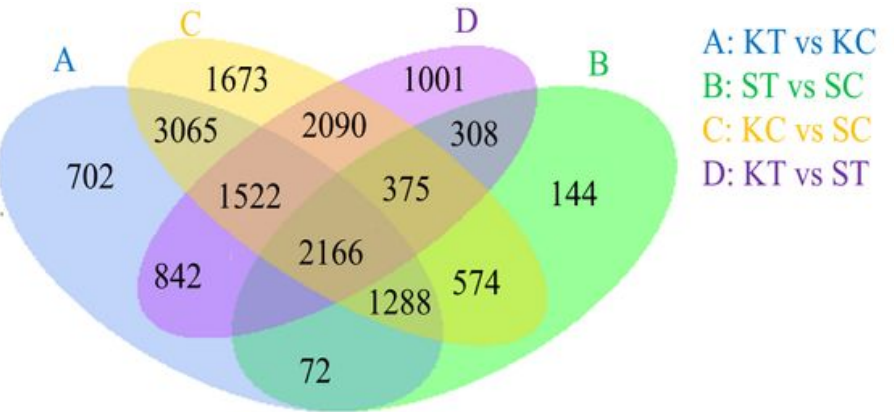

$\mathrm{C}$

KT vs KC

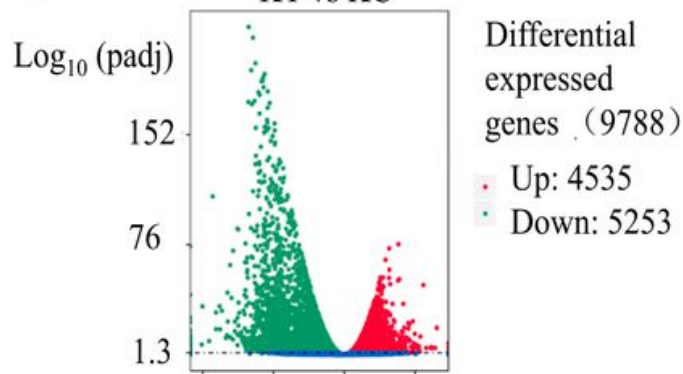

$\begin{array}{llll}-10 & -5 & 0 & 5\end{array}$

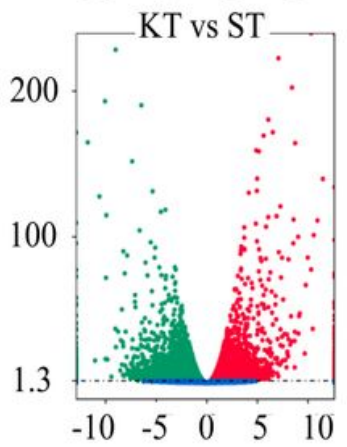

$\log _{2}$ (fold change)

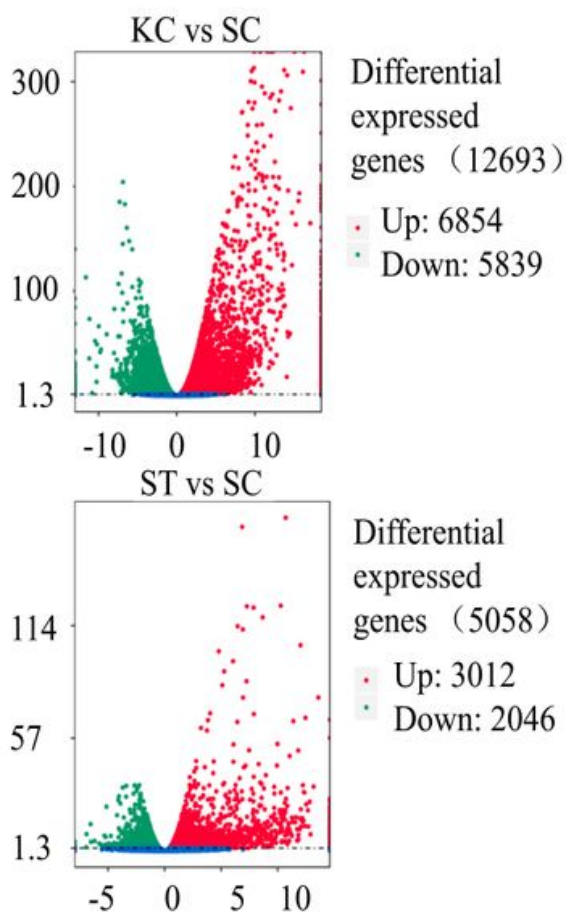

\section{Figure 3}

A: Differential gene clustering: The color ranges from red to blue, indicating log10 (FPKM+1). The blue line indicates the average relative expression of all genes in this cluster under different experimental conditions, the $\mathrm{x}$-axis indicates experimental conditions, and the $\mathrm{y}$-axis indicates relative expression. $\mathrm{B}$ : Venn diagram; Venn diagram shows the number of differential genes between the comparison groups and the overlapping relationship between the comparison groups. C: Differential Expressed genes volcano map: Genes with significant differential expression were indicated by red dots (up-regulated) and green dots (down-regulated). Genes with no significant differential expression were represented by blue dots; abscissas represent fold change of genes in different samples; ordinate represents genes Statistical significance of differences in expression changes 
A

organic sbustabce biosynthetic cellular process brological regulation bitogical process metabolic organic cyclic compound biosynthetic regulation of cellular cellular biosynthetic single-organism cullula cellular nitrogen compound biosynthetic cellular nitrogen compound metabolism organic cyclic compound metabolism gene expression

aromatic-compound biosynthetic cellular metabolic lipid metabolic heterocycle biosynthetic heterocycle metab cillecte metabolic cleobass-containing compound RNA metabolic

coenzyme bunding cofactor binding

nuclece acıd binding transeription transcription factor activity

NAD binding

The most enriched GO terms ( $\mathrm{KC}$ vs SC)

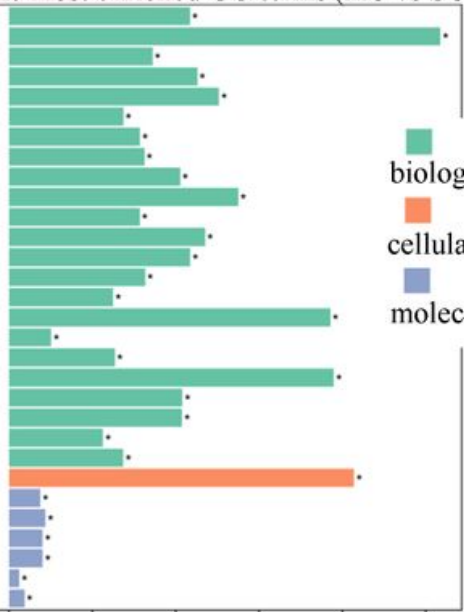

C

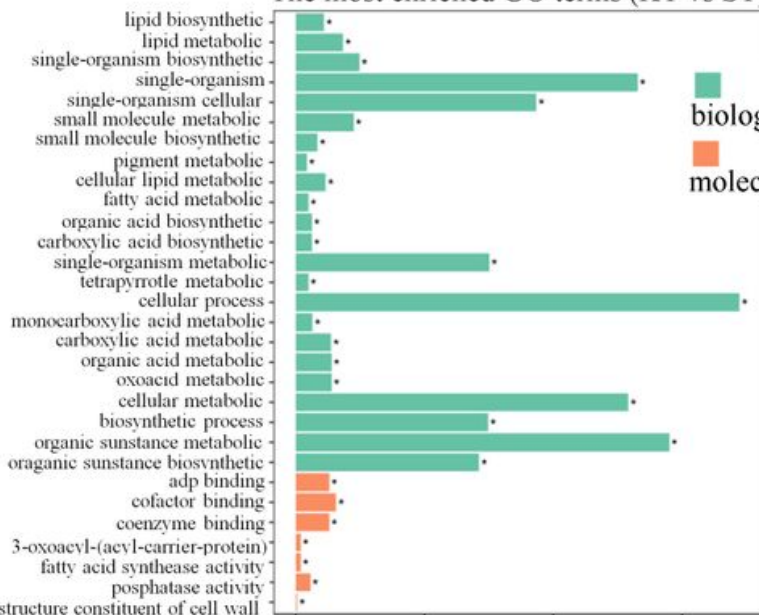

cellular process
single-organism cellula single-organism cellular ic substance biostnthetic
single-organism process organonitrogen compound metabolism
cellular biosvnthetic cellular biosvnthetic
biosynthetic process nitrogen compound metabolism . biological process single-organism metabolic small molecular metabolic earboxvlic acid metabolic

monocarboxylic acid metabolic cellular metabolic gene expression. cellular nitrogen compound organic acid metabolic oxoacid metabolic

singlelipid biosvnthetic

ribonucleoprotein complex intracellular non-membrane-bound non-membranc-bounded organelle cellular component structural constituent of ribosome 3-oxoacyl-(acyl-carrier-protein) fatty acid synthase activity cofactor binding
coenzyme binding

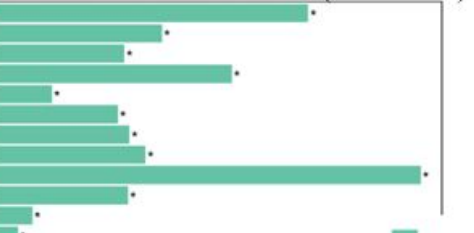

$\square$

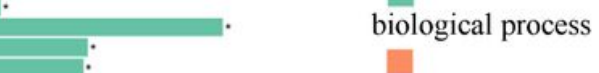

cellular component molecular function

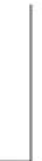

The most enriched GO terms (ST vs SC)

D

biological process organic acid biosynthetic carboxvlic acie biosvnthetic
small molecule biossnthetic nocarbosvlic acid biossnthetic lipid biosynthetic lipid metabolic
single-organism biosvnthetic single-organism process fatty acid biosynthetic metabolic process single-organism metabolic single-organism cellular carboxyc acid metabolic protem aceous extracellular matrix f prolem aceos extracellutar matrix 3-oxoacyl-(acyl-carrier-protem taity acid synthase activity transferase activit colactor bunding coenzyme binding ucleic acid binding transcription xidoreductase activity, acting loglucan: xyloglucosyl transterase transterase activity, transterase mansferase activity, transferring hexosy aroups

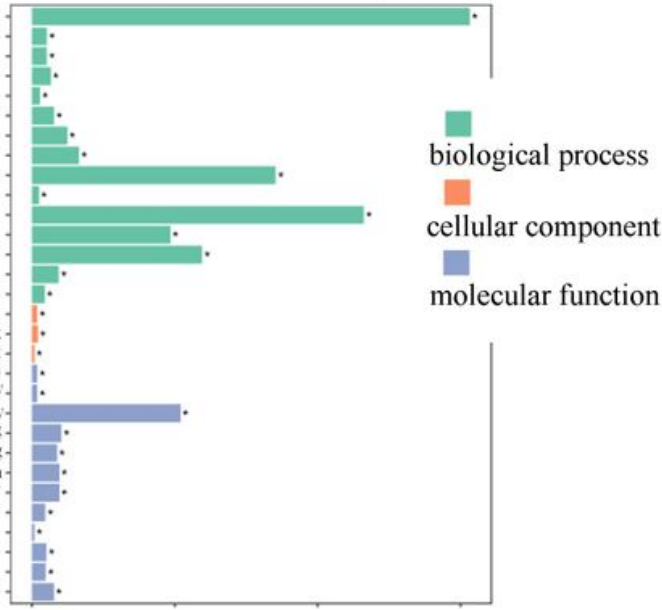

Figure 4

Differentially expressed genes in control and treated samples of Shine- Muscat and Kyoho (Biological process, Cellular component and Molecular function). 

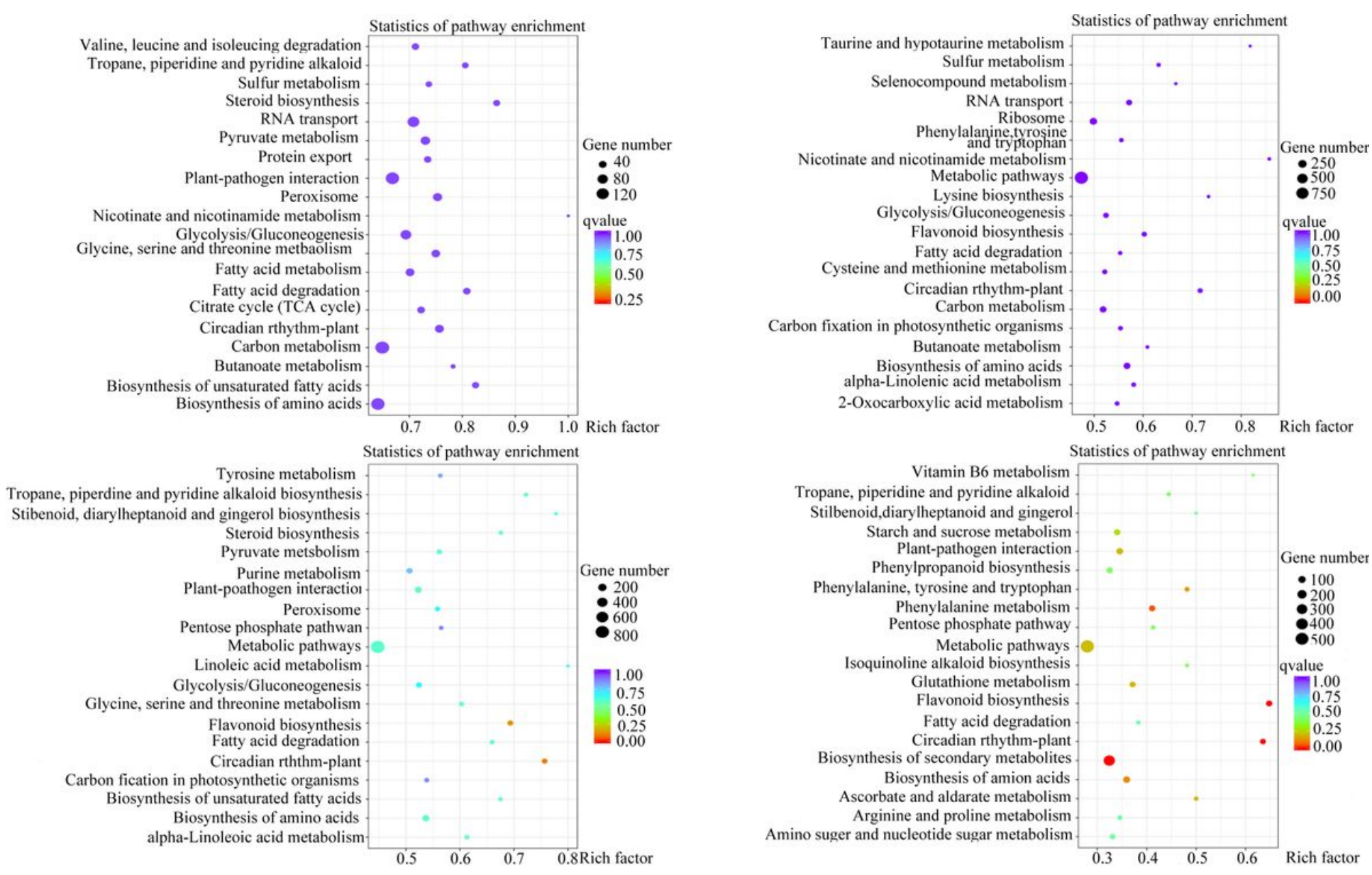

Figure 5

Differential gene KEGG enrichment scatter plot: The scatter plot is a graphical representation of the results of the KEGG enrichment analysis. In this figure, the degree of KEGG enrichment is measured by the number of genes, the Qvalue, and the number of genes enriched in this pathway. (1. KC vs SC, 2. KT vs $\mathrm{KC}, 3$. KT vs ST, 4. ST vs SC) 


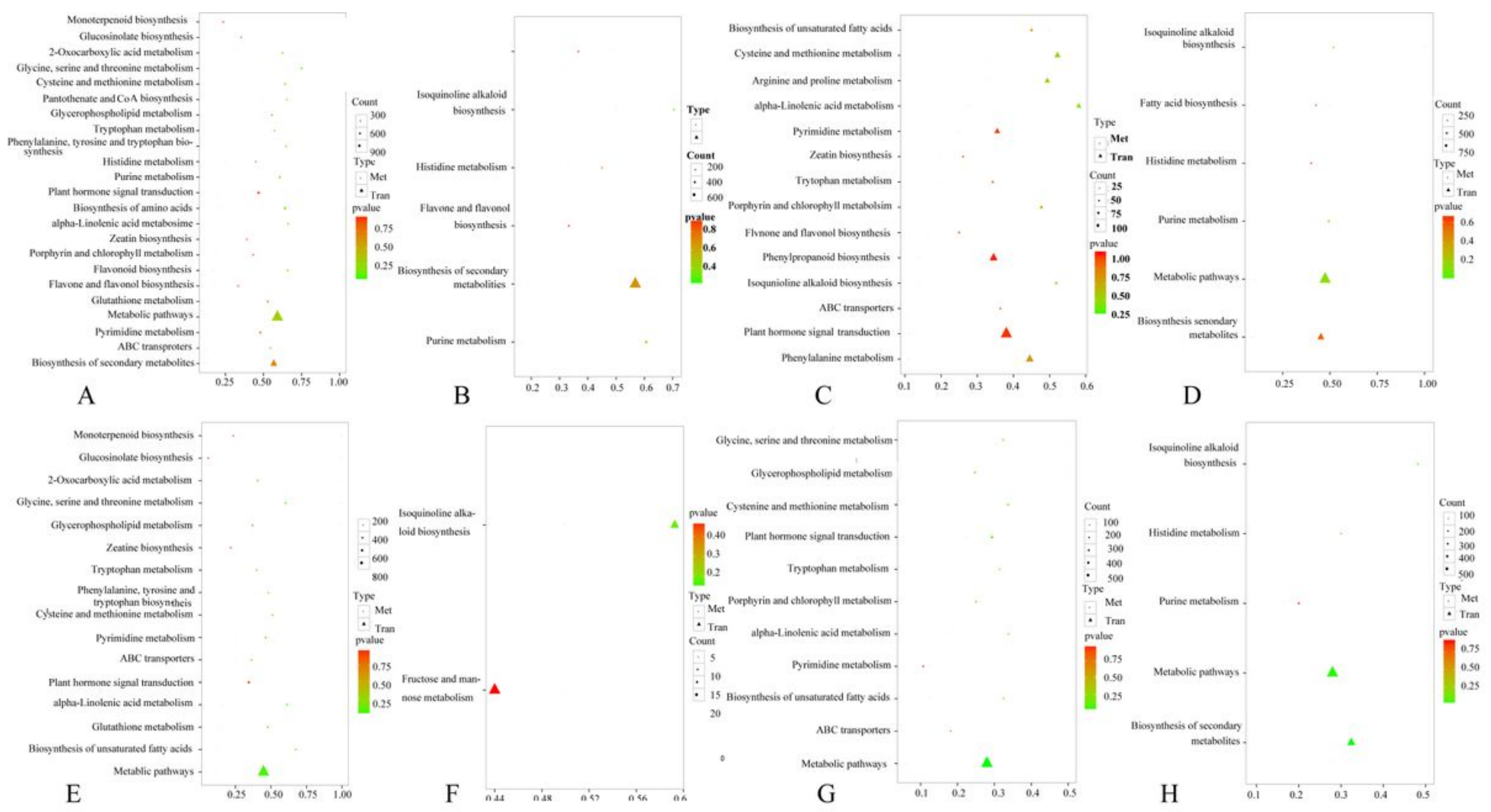

Figure 6

KEGG Enrich scatterplot; metabolism and transcription kegg enrichment comparison (KYC.vs.SHC A positive and $B$ negative; KYT.vs.KYC $C$ postive and $D$ negative; $K Y T$.vs.SHT E positiv and $F$ negative; SHT.vs.SHC G Positive and H negative)
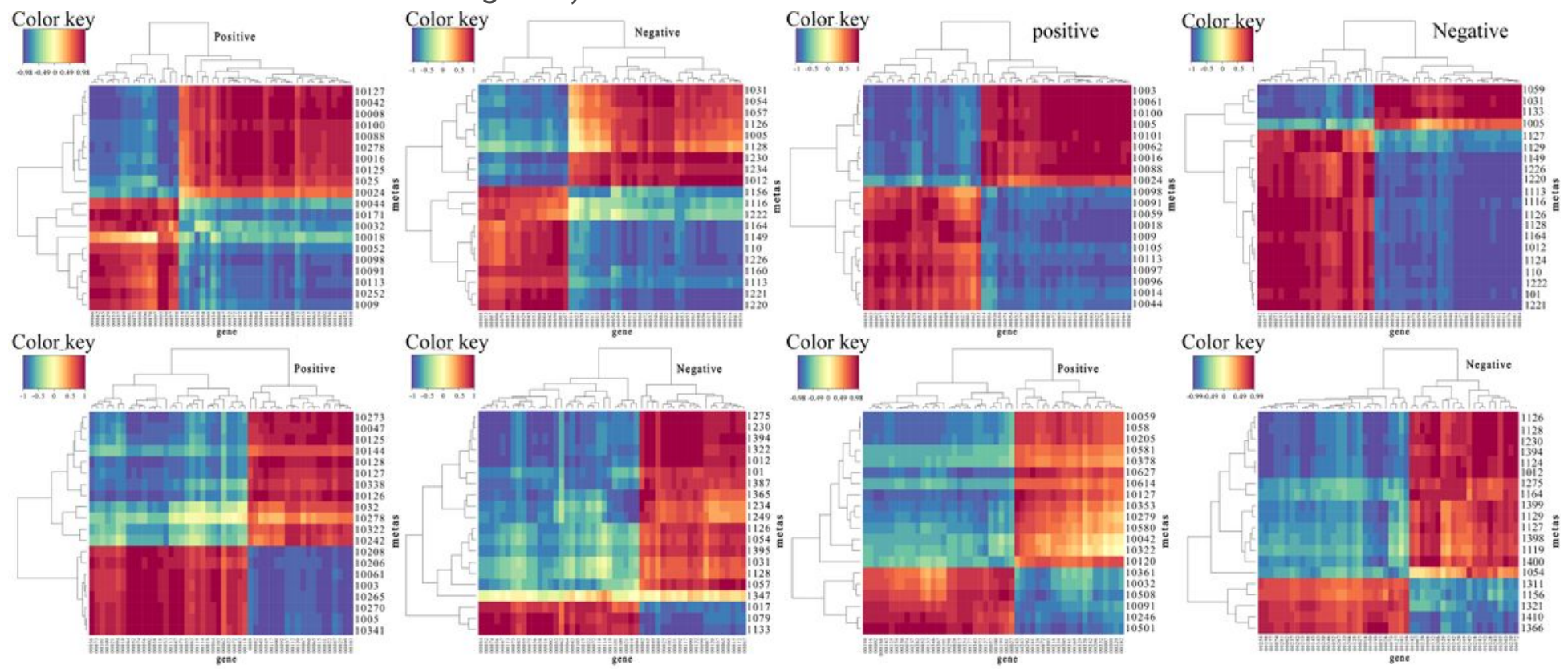

Figure 7 
The correlation analysis data of metabolites and genes, The redder the color, the stronger the positive correlation, the bluer the color, and the stronger the negative correlation. (KYC.vs.SHC A positive and B negative; KYT.vs.KYC C postive and D negative; KYT.vs.SHT E positiv and $\mathrm{F}$ negative; $\mathrm{SHT}$.vs.SHC $\mathrm{G}$ Positive and $\mathrm{H}$ negative correlation)
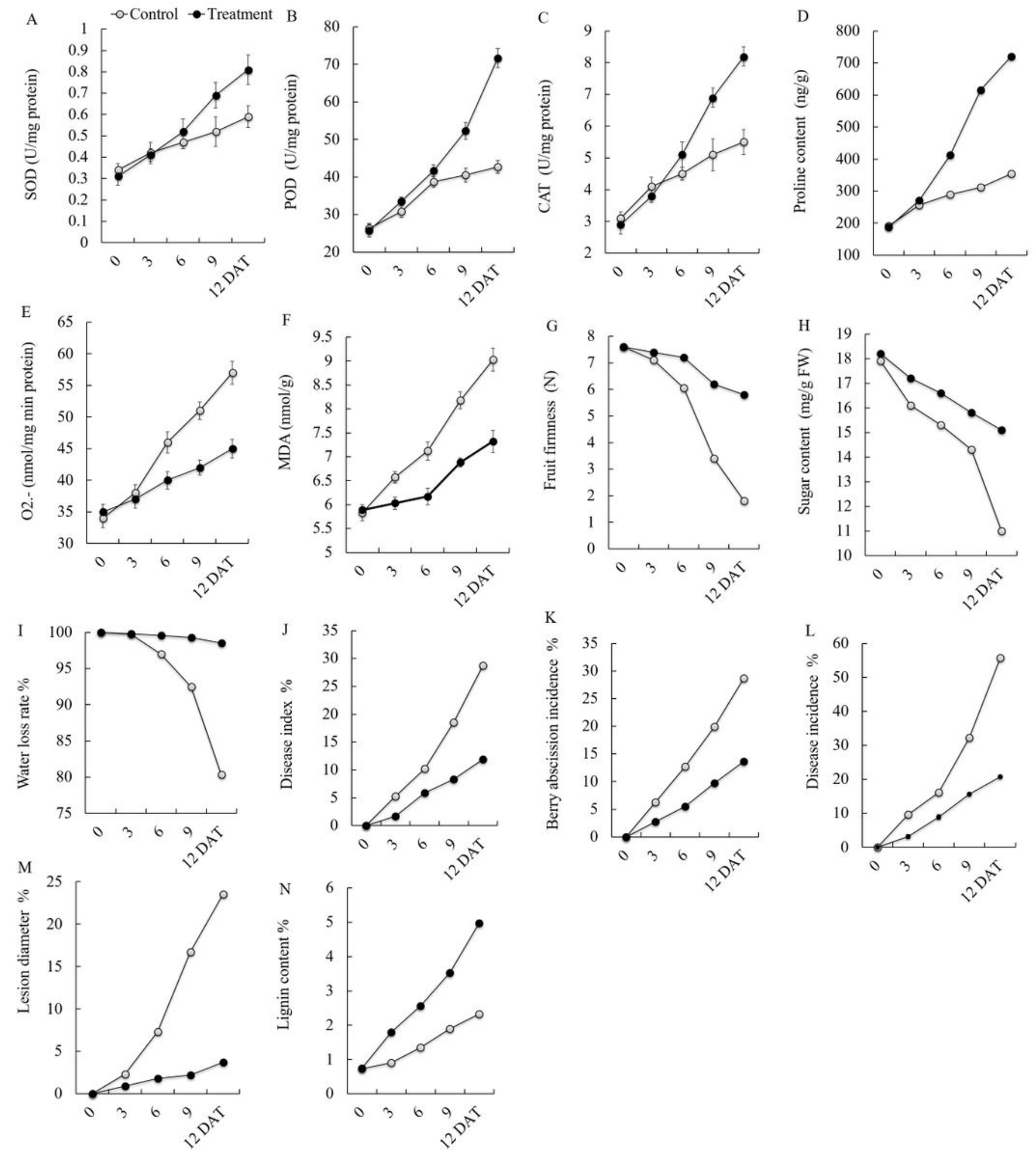

Figure 8 
Physiological and Antioxidant regulation in response to chitosan in Kyoho berries (A SOD, B POD, C CAT, D Proline content, E 02, F MDA, G Fruit firmness, H Sugar content, I Water loss rate \%age, J Disease index \%age, $\mathrm{K}$ Berry abscission incidence \% age, L Disease incidence \%age, M Lesion diameter \%age, N Lignin content \%age)
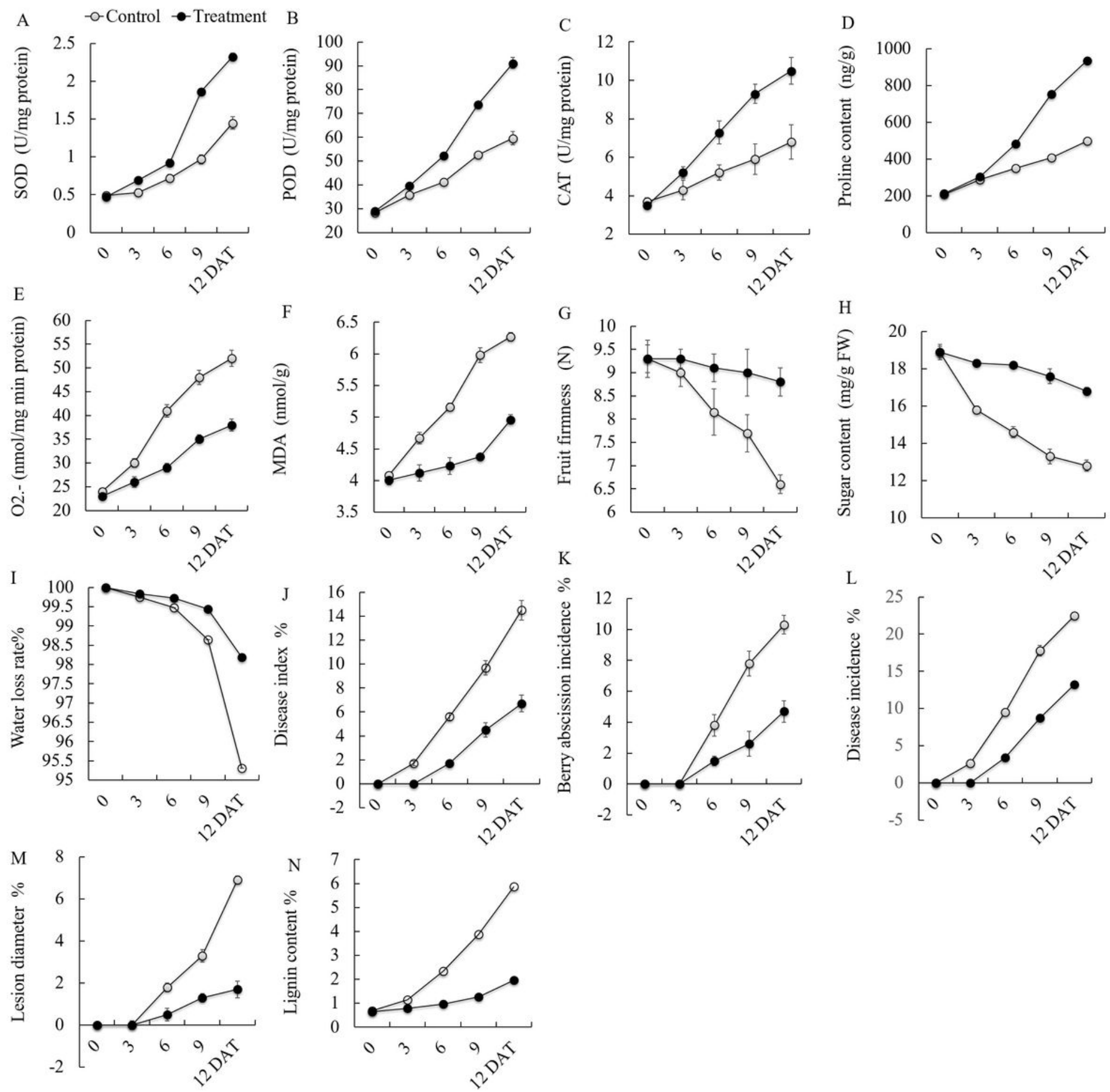

Figure 9

Physiological and Antioxidant regulation in response to chitosan in Shine-muscat berries (A SOD, B POD, C CAT, D Proline content, E 02, F MDA, G Fruit firmness, H Sugar content, I Water loss rate \%age, J Disease 
index \%age, K Berry abscission incidence \% age, L Disease incidence \%age, M Lesion diameter \%age, N Lignin content \%age)

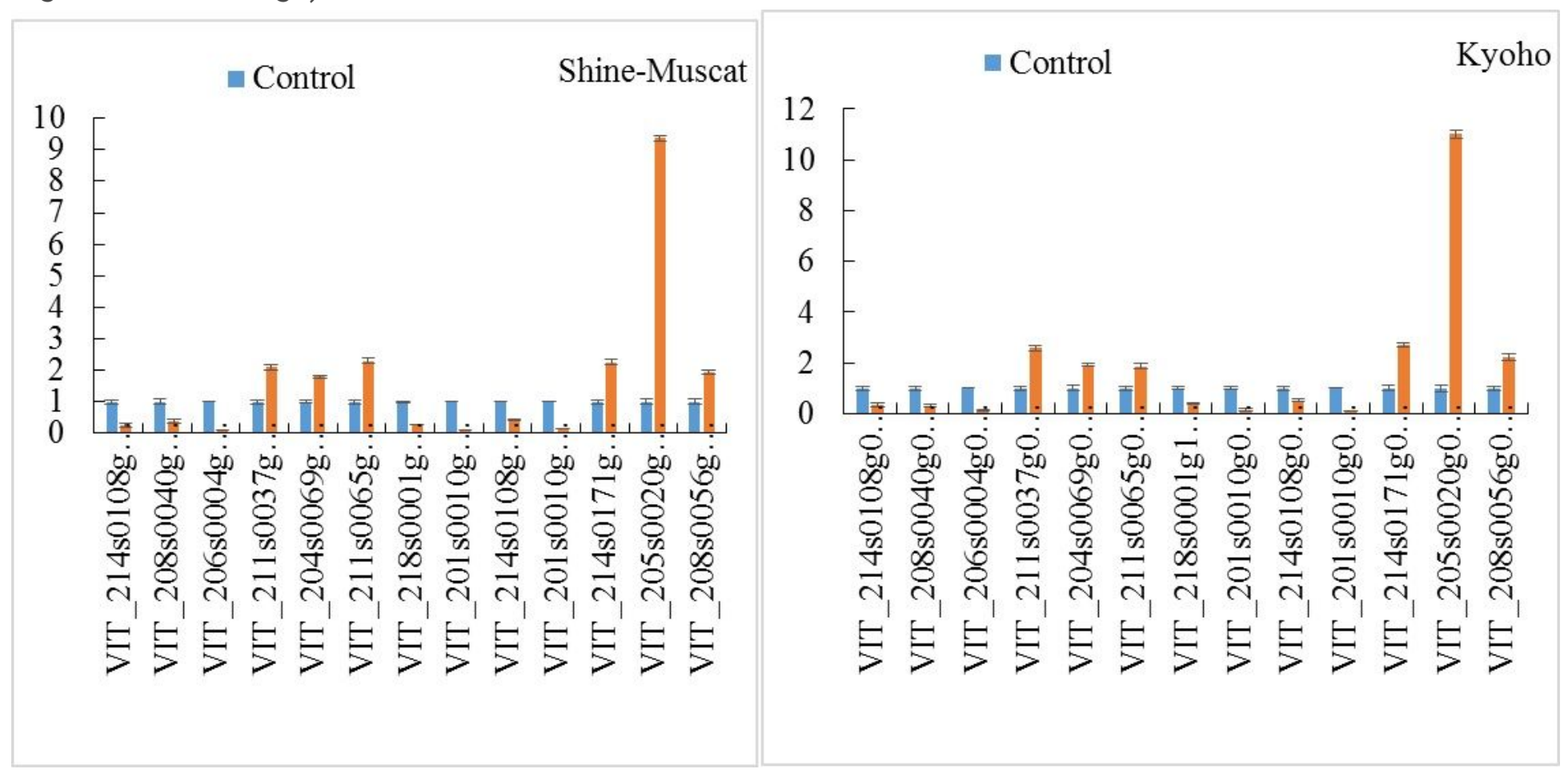

\section{Figure 10}

QRT-PCR: The validation of transcripts/genes differential expressed for 13 selected genes, measured by RNA-Seq and qRT-PCR. A; Control vs Shine-muscat and B; Control vs Kyoho.

\section{Supplementary Files}

This is a list of supplementary files associated with this preprint. Click to download.

- S3PlanthormonesignaltransductionPathway.xlsx

- S2plantpathogeninteraction.xlsx

- S5correlatoinTransciptomandmetabolom.xlsx

- S4transcriptionfactorspathwa.xlsx

- S1QrtPrimers.xIsx

- FigS1.jpg 